Review article

\title{
The dynamics of inflammatory markers in coronavirus disease-2019 (COVID-19) patients: A systematic review and meta-analysis
}

\author{
Roshan Kumar Mahat ${ }^{a, *}$, Suchismita Panda ${ }^{a}$, Vedika Rathore ${ }^{\mathrm{b}}$, Sharmistha Swain ${ }^{\mathrm{a}}$, \\ Lalendra Yadav ${ }^{\mathrm{c}}$, Sumesh Prasad Sah ${ }^{\mathrm{d}}$ \\ ${ }^{a}$ Department of Biochemistry, Pandit Raghunath Murmu Medical College and Hospital, Baripada, Mayurbhanj, Odisha, 757107, India \\ ${ }^{\mathrm{b}}$ Department of Biochemistry, Shyam Shah Medical College, Rewa, Madhya Pradesh, 486001, India \\ ${ }^{\mathrm{c}}$ Department of Pharmacology, Teerthanker Mahaveer Medical College and Research Center, Moradabad, Uttar Pradesh, 244001, India \\ d Department of Biochemistry, Muzaffarnagar Medical College, Muzaffarnagar, Uttar Pradesh, 251203, India
}

\section{A R T I C L E I N F O}

\section{Keywords:}

COVID-19

SARS-CoV-2

Coronavirus infections

Cytokine release syndrome

\begin{abstract}
A B S T R A C T
Background: Coronavirus disease-2019 (COVID-19) is a global pandemic and high mortality rate among severe or critical COVID-19 is linked with SARS-CoV-2 infection-induced hyperinflammation of the innate and adaptive immune systems and the resulting cytokine storm. This paper attempts to conduct a systematic review and metaanalysis of published articles, to evaluate the association of inflammatory parameters with the severity and mortality in COVID-19 patients.

Methods: A comprehensive systematic literature search of medical electronic databases including Pubmed/ Medline, Europe PMC, and Google Scholar was performed for relevant data published from January 1, 2020 to June 26, 2020. Observational studies reporting clear extractable data on inflammatory parameters in laboratoryconfirmed COVID-19 patients were included. Screening of articles, data extraction and quality assessment were carried out by two authors independently. Standardized mean difference (SMD)/mean difference (MD/WMD) and $95 \%$ confidence intervals (CIs) were calculated using random or fixed-effects models.

Results: A total of 83 studies were included in the meta-analysis. Of which, 54 studies were grouped by severity, 25 studies were grouped by mortality, and 04 studies were grouped by both severity and mortality. Random effect model results demonstrated that patients with severe COVID-19 group had significantly higher levels of Creactive protein (CRP), erythrocyte sedimentation rate (ESR), procalcitonin (PCT), interleukin-6 (IL-6), interleukin-10 (IL-10), interleukin-2R (IL-2R), serum amyloid A (SAA) and neutrophil-to-lymphocyte ratio (NLR) compared to those in the non-severe group. Similarly, the fixed-effect model revealed significant higher ferritin level in the severe group when compared with the non-severe group. Furthermore, the random effect model results demonstrated that the non-survivor group had significantly higher levels of CRP, PCT, IL-6, ferritin, and NLR when compared with the survivor group.

Conclusion: In conclusion, the measurement of these inflammatory parameters could help the physicians to rapidly identify severe COVID-19 patients, hence facilitating the early initiation of effective treatment.

Prospero registration number: CRD42020193169.
\end{abstract}

\section{Introduction}

Coronavirus disease 2019 (COVID-19) is caused by the zoonotic agent severe acute respiratory syndrome coronavirus 2 (SARS-CoV-2). This virus emerged in the human population in the late December 2019 in Wuhan, Hubei province, central China and has since spread across the globe. ${ }^{1,2}$ Owing to the rapid increase in the number of COVID-19 cases and uncontrolled worldwide spread, it was declared by the WHO a
Public Health Emergency of International Concern on January 30, 2020, and furthered labeled as a pandemic on March $11,2020 .^{3,4}$ As of September 28, 2020, COVID-19 pandemic had over 32.7 million confirmed cases with 991000 deaths. $^{4}$

The clinical presentation of COVID-19 ranges from mild to critically ill. While most COVID-19 patients have a mild influenza-like illness and may be asymptomatic, a minority of patients are experiencing severe pneumonia, acute respiratory distress syndrome (ARDS), multiple organ

\footnotetext{
* Corresponding author.

E-mail address: mahatroshan79@gmail.com (R.K. Mahat).
} 
failure (MOF), and even death. ${ }^{5}$ As soon as patients progress to the severity or critical stage, the risk for poor outcomes increases significantly. ${ }^{6}$ It is estimated that around $10-15 \%$ of mild COVID-19 patients advance to severe, and $15-20 \%$ of severe cases progress to become critical, with many of the individuals in the critical category needing treatment in intensive care units (ICU). ${ }^{7}$ As the number of COVID-19 cases increasing globally and treatment in intensive care units (ICU) has become a major challenge, early identification of severe forms of COVID-19 is crucial for the timely triaging of patients. ${ }^{8}$

Severe or critical COVID-19 is strongly linked with mortality ${ }^{9}$ and the high mortality rate amongst these cases is linked with SARS-CoV-2 infection-induced hyperinflammation of the innate and adaptive immune systems and the resulting cytokine storm, a cytokine release syndrome (CRS)-like syndrome in severe/critical COVID-19 cases. $^{10-13}$ Studies have reported that the inflammatory parameters are closely linked to the COVID-19 severity and mortality. ${ }^{14-17}$ In addition, two recent meta-analyses have also shown an association of inflammatory parameters with the COVID-19 severity. ${ }^{18,19}$ However, with an increase in the number of studies now published, it is important to carry out more comprehensive reviews and analyses of inflammatory parameters linked to COVID-19 severity. We, therefore, conducted a comprehensive systematic review and meta-analysis of published articles, from January 1 , 2020 to June 26, 2020, to evaluate the association of inflammatory parameters with the severity and mortality in COVID-19 patients.

\section{Methods}

This systematic review and meta-analysis has been conducted in accordance with the Preferred Reporting Items for Systematic Reviews and Meta-Analyses (PRISMA) guidelines ${ }^{20}$ and was registered with PROSPERO-The International Prospective Register of Systematic Reviews (Registration No. CRD42020193169). ${ }^{21}$

\subsection{Search strategy}

A comprehensive systematic literature search of medical electronic databases including PubMed/Medline, Europe PMC, and Google Scholar was performed for relevant data published from January 1, 2020 to June 26, 2020. Pubmed/Medline and Europe PMC were searched using the following search terms: ("COVID-19" OR "2019-nCOV" OR "SARS-COV2" OR "severe acute respiratory syndrome coronavirus 2" OR "novel coronavirus disease" OR "COVID-19 patients" OR "novel coronavirus 2019" OR "coronavirus disease-2019") AND ("erythrocyte sedimentation rate" OR "C-reactive protein" OR "ferritin" OR "procalcitonin" OR "interleukin-6" OR "interleukin-10" OR "interleukin-2R" OR "tumor necrosis factor- $\alpha$ " OR "serum amyloid A" OR "neutrophil-to-lymphocyte ratio" OR "inflammatory markers" OR "inflammatory parameters") whereas Google Scholar was searched using the keywords ("COVID-19" OR "2019-nCOV" OR "SARS-COV-2" OR "novel coronavirus disease" OR "COVID-19 patients" OR "novel coronavirus 2019" OR "coronavirus disease-2019") AND ("inflammatory markers" OR "inflammatory parameters") owing to the limitation of 256 characters in the search string. Two authors (RKM and SP) independently screened the results from the initial search by titles and abstracts for relevance and the full texts were reviewed for the eligibility criteria. To identify the eligible studies, the reference list of previous studies and systemic reviews were also searched and identified records were screened for the inclusion criteria specified for the current systemic review and meta-analysis. Any ambiguity occurred while the selection of the study was resolved by mutual discussion and consensus.

\subsection{Inclusion and exclusion criteria}

The inclusion criteria were as follows: (a) observational studies (cohort studies, case-control studies, cross-sectional studies, and case series studies) reporting clear extractable data on inflammatory parameters in laboratory-confirmed COVID-19 patients, (b) compared the inflammatory parameters between severe and non-severe COVID-19 patients or between survivors or non-survivors. The exclusion criteria were as follows: (a) review articles, non-research letters, editorials, commentaries, case reports, animal studies, original research with samples below 10, abstract from meeting proceedings, non-English language articles, (b) studies that were conducted particularly in children or pregnant women, (c) unclear reporting of levels of inflammatory parameters, (d) studies which do not provide a full-text version, (e) articles which were not peer-reviewed or accepted for publication, ( $f$ ) laboratory information not presented as mean (standard deviation, SD) or median (interquartile range, IQR or range). In addition, when two or more studies were conducted at the same center/hospital recruiting patients during the same or overlapping periods, we selected the one with a larger sample size unless the other studies presented relevant information not included in the study having a larger sample size. In this study, mild and moderate COVID-19 patients were included in the nonsevere group whereas severe and critical COVID-19 patients were included in the severe group.

\subsection{Data extraction}

Data were extracted independently by two reviewers (RKM and SP). A third reviewer (VR) checked the extracted data to ensure that there were no mistakes or duplicated information. The following information of each study was extracted from included articles: first author, country, year of publication, type of publication, hospital, date of data collection, gender, age, the total number of COVID-19 patients, number of severe/ non-severe patients, or number of survivors/non-survivors and inflammatory parameters measured.

\subsection{Quality assessment}

The quality of included studies was assessed using the NewcastleOttawa Scale (NOS) ${ }^{22}$ which is easy to use with its star rating system. Each of the included studies was judged on three broad perspectives: the selection of study groups ( $0-4$ stars), the comparability of the groups ( $0-2$ stars), and the ascertainment of the outcome of interest ( $0-3$ stars), with a maximum of nine stars representing the highest methodological quality. The quality assessment was carried out independently by two authors (RKM and SP) for each original study included. Any disagreements were discussed between the two authors, and a third author (SS) was involved, if necessary, in reaching a final judgment.

\subsection{Statistical analysis}

Mean and standard deviation of inflammatory markers were extrapolated from sample size, median and interquartile range (IQR) or range according to Luo et al. ${ }^{23}$ and Wan et al. ${ }^{24}$ when the results of the included studies were present in median and interquartile range (IQR) or range. A pooled mean difference (MD/WMD) with $95 \% \mathrm{CI}$ was used to assess the difference between inflammatory markers measured in COVID-19 patients with and without severe disease or COVID-19 patients who survived and those who did not survive in studies with the same clinical units and measures; otherwise, the standardized mean difference (SMD) was used. Statistical heterogeneity among studies was assessed using Cochran's Q test and $I^{2}$ statistics. A Cochran's $Q$ value of $<0.10$ indicates substantial heterogeneity between studies whereas $\mathrm{I}^{2}$ statistics were interpreted as $25 \%, 50 \%$, and $75 \%$ for low, moderate, and substantial heterogeneity, respectively. If heterogeneity existed, the random effect model was used; otherwise, the fixed effect model was used. Funnel plots were designed to assess the publication bias and the plot's symmetry was assessed by Egger's linear regression test (a $p$-value $<0.1$ indicated significant bias). If publication bias was confirmed, Duval and Tweedie's nonparametric trim-and-fill method was used to adjust potential publication bias. ${ }^{25} \mathrm{~A}$ leave-one-out sensitivity analysis 
was performed by removing one study at a time through influence analysis to assess the stability of results. The results of individual studies were pooled using Review Manager Version 5.4. All other statistical analyses were done using STATA (version 16; Stata Corporation, College Station, TX). A $p$-value $<0.05$ was considered statistically significant except for Egger's test and test of heterogeneity i.e. Cochran's Q test.

\section{Results}

\subsection{Outcome of the database search}

A total of 5612 articles were retrieved through the database search and from the reference lists of published articles, of which 4261 remained after the removal of duplicates. Following the screening of title/abstracts, 263 articles were selected for full-text assessment. 83 studies were finally selected for data extraction and meta-analysis after excluding ineligible studies for the following reasons: studies not stratified by severity or mortality $(\mathrm{n}=82)$, not relevant for inclusion $(\mathrm{n}=$ 10), data not extractable/unclear reporting of inflammatory parameters/data not clearly presented $(n=18)$, full text not available $(n=2)$; overlap of samples between the groups $(\mathrm{n}=1)$, inflammatory parameters not reported $(n=6)$, diagnosis not clear $(n=3)$, not laboratory diagnosed COVID-19 $(\mathrm{n}=12)$, laboratory information not presented as mean (standard deviation, SD) or median (interquartile range, IQR or range) $(n=23)$ and hospital and study period overlap with other included studies $(n=23)$. The flow diagram of the number of studies screened and included in the meta-analysis is shown in Fig. 1.

\subsection{Characteristics of the included studies and quality assessment}

We have included all the articles that were published between January 1, 2020 and June 26, 2020. All the included studies were published in the year 2020 and written in English. The main characteristics of the included studies are shown in Table 1. Of 83 articles $^{14-17}, 26-50,51-78,79-104,67$ were from China ${ }^{14-17}, 26-32$, $34-39,43-49,52-54,56-73,75-80,82,83,85,87,90,91,93,94,96,97,99-104$ and 16 were from other countries [Italy (4), ${ }^{81,84,86,92}$ Iran (2), ${ }^{51,88}$ Turkey (1), ${ }^{95}$ Korea (2), ${ }^{40,89}$ UK (1), ${ }^{98}$ Germany (1), ${ }^{33}$ Oman (1), ${ }^{41}$ Greece (1), ${ }^{42}$ Mexico City (1), ${ }^{50}$ Switzerland $(1)^{55}$ and Singapore $\left.(1)^{74}\right] .54$ articles $^{15,16,26-43,45-50,52-65,67-80}$ were grouped by severity, 25 articles $^{17,81-104}$ were grouped by mortality and 04 studies ${ }^{14,44,51,66}$ were grouped by both severity and mortality. The 58 studies which were grouped by severity contributed 10096 patients, of whom 3315 were severe COVID-19 patients and 6781 were non-severe COVID-19 patients. Among 10096 participants, 5234 were males and 4862 were females. COVID-19 severity was classified using the National Health Commission of China in 34 studies, WHO guidelines in 5 studies, American Thoracic Society guidelines for community-acquired pneumonia in 2 studies, Chinese Center for Disease Control (CDC) guidelines in 2 studies, ICU admission in 6 studies, ARDS in 2 studies, SOFA score in 1 study, the requirement of supplemental oxygen in 1 study, $\mathrm{SpO}_{2}$ in 1 study, the composite endpoint in 1 study and unspecified guidelines in 3 studies. The 29 articles which were grouped by mortality contributed 7203 patients, of whom 5644 were survivors and 1559 were patients who died of COVID-19. Among 7203 participants in the studies grouped by mortality, 3921 were males and 3282 were females. The quality of included studies was assessed based on the Newcastle-Ottawa Scale and the quality results are presented in Table 1 . Studies were awarded between 0 and 9 points, with higher scores indicating lower risk of bias.

\subsection{Metaanalysis of inflammatory markers in patients with COVID-19 stratified by severity}

Information on C-reactive protein (CRP) was available in 44 studies with 2623 severe and 5275 non-severe COVID-19 patients. The analysis of the random effect model showed that compared with the non-severe group, the severe group had a significantly higher CRP [SMD $=1.14$, 95\% CI: $0.97-1.32 ; p<0.00001]$ with a substantial heterogeneity $\left[\mathrm{I}^{2}=\right.$ 90\%]. 17 studies analyzed erythrocyte sedimentation rate (ESR) involving 1075 severe and 2362 non-severe COVID-19 patients. The value of ESR was significantly higher in the severe group when compared with the non-severe group [MD $=12.08$, 95\% CI: 8.04-16.11; $p<0.00001]$ with a high heterogeneity $\left[\mathrm{I}^{2}=75 \%\right]$ in a random effect model. 30 studies with 2217 severe and 3682 non-severe COVID-19 patients were included for the meta-analysis of procalcitonin (PCT). The random effect model demonstrated that the severe group had significantly increased PCT than the non-severe group $[\mathrm{SMD}=0.88,95 \% \mathrm{CI}$ : $0.68-1.08 ; p<0.00001]$ with a substantial heterogeneity $\left[\mathrm{I}^{2}=90 \%\right]$. A total of 18 studies with 1564 severe and 2054 non-severe COVID-19 patients were included in this metaanalysis for interleukin-6 (IL-6). In a random effect model, the value of IL-6 was significantly higher in the severe group when compared with the non-severe group $[\mathrm{MD}=16.94$, 95\% CI: $12.72-21.16 ; p<0.00001]$ with a substantial heterogeneity [I ${ }^{2}$ $=96 \%$ ]. In total, 8 studies with 864 severe and 762 non-severe COVID19 patients were taken in the metaanalysis for interleukin-10 (IL-10). The estimated pooled MD indicated that the severe group had a significantly higher level of IL-10 than the non-severe group [MD $=2.03,95 \%$ CI: $1.36-2.70 ; p<0.00001]$ with a substantial heterogeneity $\left[\mathrm{I}^{2}=82 \%\right]$ in a random effect model. Information on interleukin-2R (IL-2R) was available in two studies, including 345 severe and 186 non-severe COVID-19 patients. In a random effect model, the value of IL-2R was significantly higher in the severe group when compared with the nonsevere group [MD $=238.26$, 95\% CI: 31.90-444.62; $p=0.02]$ with high heterogeneity $\left[\mathrm{I}^{2}=84 \%\right]$. For tumor necrosis factor- $\alpha$ (TNF- $\alpha$ ), 7 studies with 758 severe and 682 non-severe COVID-19 were included in the meta-analysis and the random effect model analysis showed that compared with the non-severe group, the severe group had higher TNF$\alpha$, but the difference was not significant $[\mathrm{MD}=0.05,95 \% \mathrm{CI}$ : $-0.57-0.68$; $\left.p=0.87, \mathrm{I}^{2}=91 \%\right]$. We obtained information about ferritin from 9 studies including 835 severe and 774 non-severe COVID-19 patients. The estimated pooled standardized mean difference indicated that the severe group had significantly higher ferritin than the non-severe group [SMD $=0.71,95 \%$ CI: $0.60-0.81 ; p<0.00001]$ without evident heterogeneity $\left[I^{2}=5 \%\right]$ in a fixed-effect model. Nine studies with 482 severe and 807 non-severe COVID-19 patients were included in the metaanalysis for serum amyloid A (SAA). The estimated pooled standardized mean difference revealed a significant increase in SAA in the severe group compared with the non-severe group [SMD $=1.16,95 \% \mathrm{CI}$ : $0.64-1.68 ; p<0.0001]$ with a substantial heterogeneity $\left[\mathrm{I}^{2}=93 \%\right]$ in a random effect model. The meta-analysis included 819 severe and 1700 non-severe COVID-19 patients from 12 studies for neutrophil-tolymphocyte ratio (NLR) and a random effect model analysis revealed significant higher NLR in severe patients when compared with nonsevere COVID-19 patients [MD $=3.27,95 \%$ CI: $1.99-4.55 ; p<$ $0.00001]$ with a substantial heterogeneity $\left[\mathrm{I}^{2}=90 \%\right]$ (Table 2; Supplement 1).

\subsection{Metaanalysis of inflammatory markers in patients with COVID-19 stratified by mortality}

We obtained information about CRP from 19 studies including 3427 survivors and 891 non-survivors. Random effect model analysis showed significantly increased CRP in the non-survivor group when compared to the survivor group [SMD $=1.18,95 \%$ CI: $0.80-1.55 ; p<0.00001]$ with a substantial heterogeneity $\left[\mathrm{I}^{2}=94 \%\right]$. Seven studies with 815 survivors and 347 non-survivors with COVID-19 were included in the metaanalysis for ESR. The non-survivor group had an insignificant increase in the level of ESR compared with the survivor group [MD $=12.98,95 \%$ CI: -1.79-27.75; $p=0.08]$ with a substantial heterogeneity $\left[\mathrm{I}^{2}=91 \%\right]$ in a random effect model. Information on PCT was available in 12 studies with 2260 survivors and 762 non-survivors. The pooled median difference revealed a significant increase in the level of PCT in the non- 


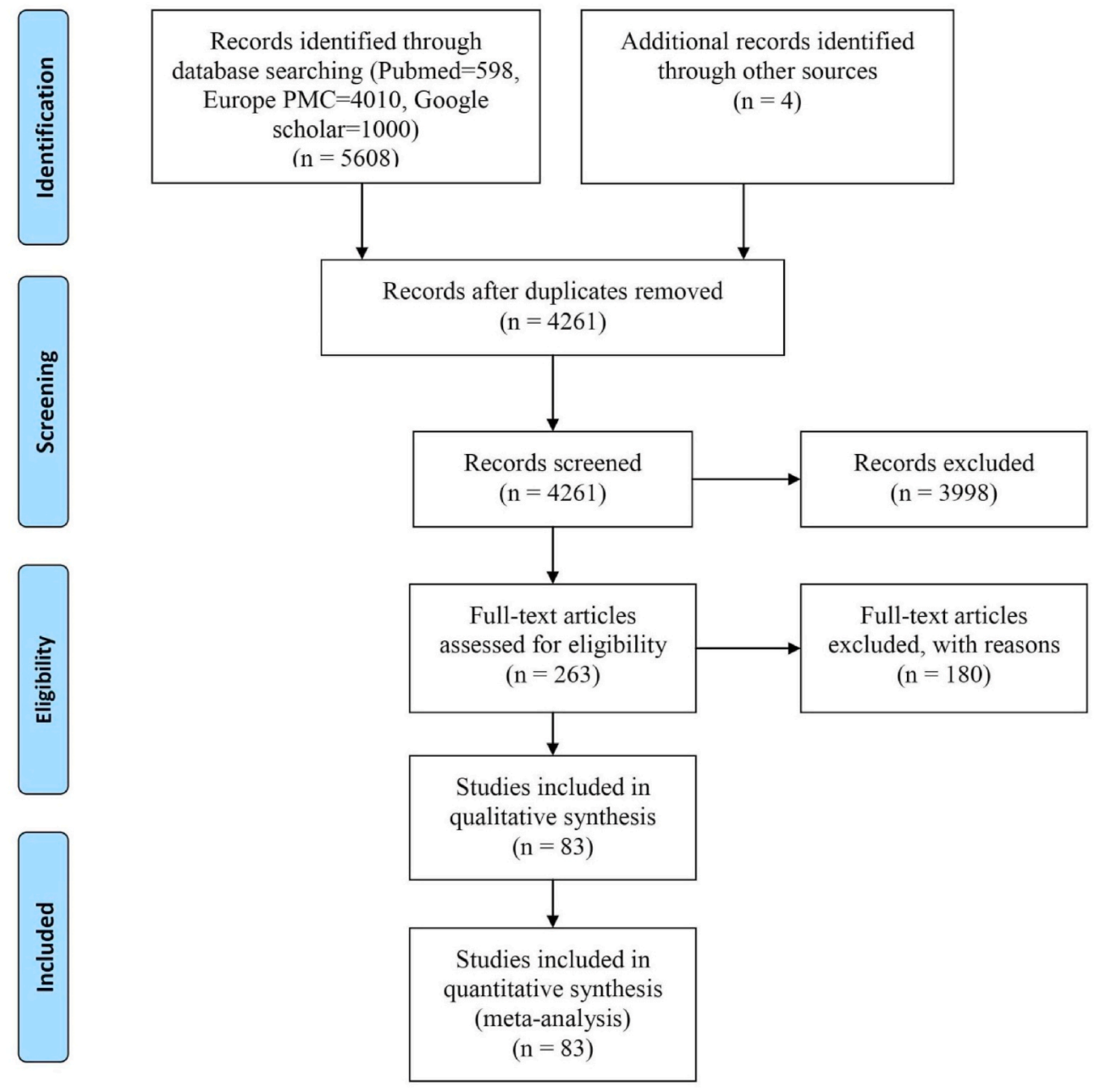

Fig. 1. PRISMA flow chart of the study selection procedure.

survivor group compared with the survivor group $[\mathrm{MD}=0.26,95 \% \mathrm{CI}$ : $0.18-0.34 ; p<0.00001]$ with a high heterogeneity $\left[\mathrm{I}^{2}=85 \%\right]$ in a random effect model. For IL-6, eight studies involving 1478 survivors and 587 non-survivors were included in the meta-analysis. The random effect model demonstrated a significantly higher level of IL- 6 in nonsurvivors than the survivors [MD $=15.62,95 \% \mathrm{CI}$ : $10.67-20.57 ; p<$ $0.00001]$ with a substantial heterogeneity $\left[\mathrm{I}^{2}=96 \%\right]$. A total of 11 studies with 1904 survivors and 650 non-survivors were included in the present meta-analysis for ferritin. In a random effect model, the value of ferritin was significantly higher in the non-survivor group when compared with the survivor group [SMD $=0.95,95 \%$ CI: 0.74-1.17; $p<$ $0.00001]$ with a high heterogeneity $\left[\mathrm{I}^{2}=76 \%\right]$. Seven studies analyzed NLR involving 2068 survivors and 282 non-survivors. The non-survivor group had a significantly increased level of NLR when compared with survivor [MD $=8.96,95 \%$ CI: $3.97-13.95 ; p=0.0004]$ with a high heterogeneity $\left[\mathrm{I}^{2}=99 \%\right]$ in a random effect model (Table 3; Supplement 2). IL-10, IL-2R, and TNF- $\alpha$ were not included in the meta-analysis since the information about these parameters were available in only one study. In addition, information about SAA was also available in only one study and hence this was also not included in the meta-analysis.

\subsection{Subgroup analysis}

In a subgroup analysis by sample size, we did not find any significant differences in the levels of CRP, ESR, PCT, ferritin, SAA, NLR, IL-10, and TNF- $\alpha$ between the sample size $\geq 100$ subgroup and sample size $<100$ subgroup. However, IL-6 was significantly increased in sample size
$<100$ subgroup compared with the sample size $\geq 100$ subgroup. In both the subgroups, CRP, ESR, PCT, ferritin, SAA, NLR, IL-10, IL-6, and TNF- $\alpha$ were associated with the severity of COVID-19 (Supplement 3).

Subgroup analysis based on sample size showed a significant association of CRP, ferritin, and NLR with mortality of COVID-19 patients in both the sample size $<100$ subgroup and sample size $\geq 100$ subgroup, whereas PCT was significantly associated with mortality in sample size $\geq 100$ subgroup only. In neither of the subgroup, ESR was significantly associated with mortality. In addition, we did not find any significant differences in the levels of CRP, ESR, PCT, and ferritin between the sample size $\geq 100$ subgroup and sample size $<100$ subgroup. However, the sample size $\geq 100$ subgroup had a significantly increased level of NLR when compared with sample size $<100$ subgroup (Supplement 4).

\subsection{Publication bias}

Funnel plots were constructed for only those parameters which were retrieved from $\geq 10$ studies. Funnel plot analysis showed asymmetrical shape for CRP, ESR, PCT, IL6, and NLR in severity studies (Supplement $5)$. Regression-based Egger's test showed statistically significant smallstudy effects for CRP $(p=0.0000)$, ESR $(p=0.0802)$, PCT ( $p=$ $0.0531)$, IL-6 $(p=0.0000)$ and NLR $(p=0.0085)$. Therefore, to adjust the publication bias, the trim and fill method was adopted and after adjustment, the funnel plot looks more symmetric than before. The trim and fill method did not impute any study in CRP, PCT, and NLR whereas 5 and 6 studies were imputed in ESR and IL-6 respectively (Supplement 6). 
Table 1

Characteristics of included studies.

\begin{tabular}{|c|c|c|c|c|c|c|c|c|c|c|c|c|}
\hline \multicolumn{13}{|c|}{ Studies stratified by severity } \\
\hline Author & Country & $\begin{array}{l}\text { Year of } \\
\text { publication }\end{array}$ & Hospitals & $\begin{array}{l}\text { Type of } \\
\text { publication }\end{array}$ & $\begin{array}{l}\text { Date of data } \\
\text { collection }\end{array}$ & $\begin{array}{l}\text { Gender } \\
(\mathrm{M} / \mathrm{F})\end{array}$ & $\begin{array}{l}\text { Total } \\
\text { patients }\end{array}$ & $\begin{array}{l}\text { Non- } \\
\text { severe } \\
\text { patients }\end{array}$ & $\begin{array}{l}\text { Severe } \\
\text { patients }\end{array}$ & $\begin{array}{l}\text { Age, median (IQR) } \\
\text { or mean } \pm \mathrm{SD}\end{array}$ & $\begin{array}{l}\text { Parameters } \\
\text { extracted }\end{array}$ & NOS \\
\hline Cao $\mathrm{Z}$ et al. ${ }^{26}$ & China & 2020 & Beijing You'an Hospital & $\begin{array}{l}\text { Retrospective } \\
\text { study }\end{array}$ & $\begin{array}{l}21 \text { January to } \\
12 \text { February, } \\
2020\end{array}$ & $38 / 42$ & 80 & 53 & 27 & $53 \pm 20$ & CRP, PCT & 7 \\
\hline Cen $\mathrm{Y}$ et al..$^{27}$ & China & 2020 & $\begin{array}{l}\text { Huoshenshan Hospital, General } \\
\text { Hospital of the Central Theatre } \\
\text { Command of the People's Liberation } \\
\text { Army, and mobile cabin hospitals in } \\
\text { Wuhan }\end{array}$ & $\begin{array}{l}\text { Observational } \\
\text { Cohort study }\end{array}$ & $\begin{array}{l}\text { As of } 10 \\
\text { February } \\
2020\end{array}$ & $493 / 514$ & 1007 & 720 & 287 & $61(49-68)$ & CRP, PCT & 8 \\
\hline Chen Cet al. ${ }^{28}$ & China & 2020 & Third People's Hospital of Shenzhen & $\begin{array}{l}\text { Retrospective } \\
\text { study }\end{array}$ & $\begin{array}{l}11 \text { January to } \\
18 \text { February, } \\
2020 .\end{array}$ & $198 / 219$ & 417 & 325 & 92 & $47(34-60)$ & $\begin{array}{l}\text { CRP, PCT, IL-6, } \\
\text { ESR }\end{array}$ & 8 \\
\hline $\begin{array}{l}\text { Chen } \mathbf{Q} \\
\text { et al. }\end{array}$ & China & 2020 & $\begin{array}{l}\text { Taizhou Public Health Medical } \\
\text { Center }\end{array}$ & $\begin{array}{l}\text { Retrospective } \\
\text { study }\end{array}$ & $\begin{array}{l}1 \text { January to } \\
11 \text { March, } \\
2020\end{array}$ & $79 / 66$ & 145 & 102 & 43 & $47.5 \pm 14.6$ & CRP, ESR, PCT & 7 \\
\hline $\begin{array}{l}\text { Chen } \mathrm{R} \\
\text { et al. }{ }^{14}\end{array}$ & China & 2020 & 575 hospitals throughout China & $\begin{array}{l}\text { Retrospective } \\
\text { study }\end{array}$ & $\begin{array}{l}\text { By March 22, } \\
2020\end{array}$ & $313 / 235$ & 548 & 345 & 203 & $56.0 \pm 14.5$ & $\begin{array}{l}\text { CRP, PCT, IL-6, } \\
\text { SAA, NLR, } \\
\text { Ferritin }\end{array}$ & 7 \\
\hline Chen $X$ et al. ${ }^{30}$ & China & 2020 & $\begin{array}{l}\text { General Hospital of Central Theater } \\
\text { Command, PLA }\end{array}$ & $\begin{array}{l}\text { Retrospective } \\
\text { study }\end{array}$ & $\begin{array}{l}\text { February } 1 \text { to } \\
\text { February } 19 \\
2020\end{array}$ & $37 / 11$ & 48 & 21 & 27 & $64.6 \pm 18.1$ & PCT, IL-6 & 6 \\
\hline Ding $X$ et al. ${ }^{31}$ & China & 2020 & Beijing YouAn Hospital & $\begin{array}{l}\text { Retrospective } \\
\text { study }\end{array}$ & $\begin{array}{l}21 \text { January to } \\
17 \text { February, } \\
2020\end{array}$ & $33 / 39$ & 72 & 57 & 15 & $49(37-64)$ & NLR & 8 \\
\hline $\begin{array}{l}\text { Dong Y } \\
\text { et al. }{ }^{32}\end{array}$ & China & 2020 & Union Hospital & $\begin{array}{l}\text { Retrospective } \\
\text { study }\end{array}$ & $\begin{array}{l}10 \text { February } \\
\text { to } 29 \\
\text { February, } \\
2020\end{array}$ & $63 / 84$ & 147 & 94 & 53 & $48(36-62)$ & ESR, SAA & 7 \\
\hline $\begin{array}{l}\text { Dreher M } \\
\text { et al. }^{33}\end{array}$ & Germany & 2020 & Aachen University Hospital & $\begin{array}{l}\text { Retrospective } \\
\text { study }\end{array}$ & $\begin{array}{l}\text { February to } \\
\text { March } 2020\end{array}$ & $33 / 17$ & 50 & 26 & 24 & $65(58-76)$ & CRP, PCT, IL-6 & 8 \\
\hline Duan J et al. ${ }^{34}$ & China & 2020 & $\begin{array}{l}\text { Chongqing Three Gorges Central } \\
\text { Hospital and Chongqing Public } \\
\text { Health Medical Center }\end{array}$ & $\begin{array}{l}\text { Retrospective } \\
\text { study }\end{array}$ & $\begin{array}{l}1 \text { January to } \\
29 \text { th } \\
\text { February, } \\
2020\end{array}$ & $184 / 164$ & 348 & 328 & 20 & $\begin{array}{l}\text { Non-severe: } 44 \pm \\
\text { 15; Severe: } 58 \pm 15\end{array}$ & CRP, PCT, NLR & 8 \\
\hline Feng Y et al. ${ }^{35}$ & China & 2020 & $\begin{array}{l}\text { Jinyintan Hospital in Wuhan, } \\
\text { Shanghai Public Health Clinical } \\
\text { Center in Shanghai, and Tongling } \\
\text { People's Hospital in Anhui Province }\end{array}$ & $\begin{array}{l}\text { Retrospective } \\
\text { study }\end{array}$ & $\begin{array}{l}1 \text { January to } \\
15 \text { February, } \\
2020\end{array}$ & $271 / 205$ & 476 & 352 & 124 & $53(40-64)$ & CRP, PCT, ESR & 8 \\
\hline $\mathrm{Fu} \mathrm{J}$ et al. ${ }^{36}$ & China & 2020 & $\begin{array}{l}\text { Affiliated Infectious Diseases } \\
\text { Hospital of Soochow University }\end{array}$ & $\begin{array}{l}\text { Retrospective } \\
\text { study }\end{array}$ & $\begin{array}{l}20 \text { January to } \\
20 \text { February, } \\
2020\end{array}$ & $45 / 30$ & 75 & 59 & 16 & $46.6 \pm 14$ & CRP, PCT, NLR & 8 \\
\hline Gao Y et al. ${ }^{37}$ & China & 2020 & $\begin{array}{l}\text { Fuyang Second People's } \\
\text { Hospital }\end{array}$ & $\begin{array}{l}\text { Retrospective } \\
\text { study }\end{array}$ & $\begin{array}{l}23 \text { January to } \\
2 \text { February, } \\
2020\end{array}$ & $26 / 17$ & 43 & 28 & 15 & $43.74 \pm 12.12$ & CRP, PCT, IL-6 & 7 \\
\hline Gong $\mathrm{J}$ et al. ${ }^{38}$ & China & 2020 & $\begin{array}{l}\text { Guangzhou Eighth People's Hospital, } \\
\text { Zhongnan Hospital of Wuhan } \\
\text { University and the Third Affiliated } \\
\text { Hospital of Sun Yat-sen University } \\
\text { but } 189 \text { used in the analysis come } \\
\text { only from Guangzhou Eighth } \\
\text { People's Hospital }\end{array}$ & $\begin{array}{l}\text { Retrospective } \\
\text { study }\end{array}$ & $\begin{array}{l}20 \text { January to } \\
2 \text { March, } \\
2020\end{array}$ & $88 / 101$ & 189 & 161 & 28 & $49(35-63)$ & SAA, NLR & 8 \\
\hline
\end{tabular}




\begin{tabular}{|c|c|c|c|c|c|c|c|c|c|c|c|c|}
\hline $\begin{array}{l}\text { Huang C } \\
\text { et al. }^{39}\end{array}$ & China & 2020 & Jin Yintan Hospital & $\begin{array}{l}\text { Prospective } \\
\text { study }\end{array}$ & $\begin{array}{l}16 \text { December } \\
2019 \text { to } 2 \\
\text { January, } \\
2020\end{array}$ & $30 / 11$ & 41 & 28 & 13 & $49(41-58)$ & PCT & 7 \\
\hline $\begin{array}{l}\text { Jang JG } \\
\text { et al. }{ }^{40}\end{array}$ & Korea & 2020 & $\begin{array}{l}\text { Yeungnam University Medical } \\
\text { Center }\end{array}$ & $\begin{array}{l}\text { Retrospective } \\
\text { study }\end{array}$ & $\begin{array}{l}19 \text { February } \\
\text { to } 15 \text { April, } \\
2020\end{array}$ & $48 / 62$ & 110 & 87 & 23 & $56.9 \pm 17.0$ & CRP, PCT & 8 \\
\hline $\begin{array}{l}\text { Khamis F } \\
\text { et al. }^{41}\end{array}$ & $\begin{array}{l}\text { Muscat } \\
\text { Oman }\end{array}$ & 2020 & $\begin{array}{l}\text { The Royal Hospital and Al Nahdha } \\
\text { Hospital }\end{array}$ & A case series & $\begin{array}{l}24 \text { February } \\
\text { to } 24 \text { April, } \\
2020\end{array}$ & $53 / 10$ & 63 & 39 & 24 & $48 \pm 16$ & CRP, Ferritin & 7 \\
\hline $\begin{array}{l}\text { Lagadinou M } \\
\text { et al. }\end{array}$ & Greece & 2020 & Patras University Hospital & $\begin{array}{l}\text { Retrospective } \\
\text { study }\end{array}$ & $\begin{array}{l}4 \text { March to } 4 \\
\text { April, } 2020\end{array}$ & $31 / 33$ & 64 & 16 & 48 & $57.11 \pm 16.3$ & $\begin{array}{l}\text { CRP, Ferritin, } \\
\text { NLR }\end{array}$ & 6 \\
\hline Li H et al. ${ }^{43}$ & China & 2020 & Tianyou Hospital & $\begin{array}{l}\text { Retrospective } \\
\text { study }\end{array}$ & $\begin{array}{l}18 \text { January to } \\
26 \text { February, } \\
2020\end{array}$ & $75 / 57$ & 132 & 60 & 72 & $62.05 \pm 12.68$ & CRP, PCT, SAA & 8 \\
\hline Li J et al. ${ }^{44}$ & China & 2020 & Central Hospital of Wuhan & $\begin{array}{l}\text { Retrospective } \\
\text { study }\end{array}$ & $\begin{array}{l}1 \text { January to } \\
20 \text { February, } \\
2020\end{array}$ & $75 / 59$ & 134 & 45 & 89 & $\begin{array}{l}61.00 \\
(46.75-69.25)\end{array}$ & $\begin{array}{l}\text { CRP, PCT, ESR, } \\
\text { IL-6, Ferritin }\end{array}$ & 7 \\
\hline Li K et al. ${ }^{45}$ & China & 2020 & $\begin{array}{l}\text { The Second Affiliated } \\
\text { Hospital of Chongqing Medical } \\
\text { University }\end{array}$ & $\begin{array}{l}\text { Retrospective } \\
\text { study }\end{array}$ & $\begin{array}{l}\text { January } 2020 \\
\text { to February } \\
2020\end{array}$ & $44 / 39$ & 83 & 58 & 25 & $45.5 \pm 12.3$ & CRP, PCT & 7 \\
\hline Liu J et al. ${ }^{46}$ & China & 2020 & Union Hospital & $\begin{array}{l}\text { Retrospective } \\
\text { study }\end{array}$ & $\begin{array}{l}5 \text { January to } \\
24 \text { January, } \\
2020\end{array}$ & $15 / 25$ & 40 & 27 & 13 & $48.7 \pm 13.9$ & SAA & 7 \\
\hline Liu $\mathrm{T}$ et al. ${ }^{47}$ & China & 2020 & Union Hospital & $\begin{array}{l}\text { Retrospective } \\
\text { study }\end{array}$ & $\begin{array}{l}21 \text { January to } \\
16 \text { February, } \\
2020\end{array}$ & $34 / 46$ & 80 & 11 & 69 & $\begin{array}{l}53.00 \text { (Range: } \\
26.00-86.00)\end{array}$ & Ferritin & 6 \\
\hline Lo IL et al. ${ }^{48}$ & China & 2020 & $\begin{array}{l}\text { Centro Hospitalar Conde de São } \\
\text { Januário (C.H.C.S.J.) }\end{array}$ & $\begin{array}{l}\text { Retrospective } \\
\text { study }\end{array}$ & $\begin{array}{l}21 \text { January to } \\
16 \text { February, } \\
2020\end{array}$ & $3 / 7$ & 10 & 6 & 4 & $54(27-64)$ & CRP & 7 \\
\hline Lv Z et al. ${ }^{16}$ & China & 2020 & $\begin{array}{l}\text { Renmin Hospital of Wuhan } \\
\text { University }\end{array}$ & $\begin{array}{l}\text { Retrospective } \\
\text { study }\end{array}$ & $\begin{array}{l}4 \text { February to } \\
28 \text { February, } \\
2020\end{array}$ & $175 / 179$ & 354 & 115 & 239 & 62 (Range: $23-90)$ & $\begin{array}{l}\text { CRP, PCT, IL-6, } \\
\text { IL-10, TNF- } \alpha\end{array}$ & 7 \\
\hline Ma J et al. ${ }^{49}$ & China & 2020 & $\begin{array}{l}\text { Renmin Hospital of Wuhan } \\
\text { University }\end{array}$ & $\begin{array}{l}\text { Retrospective } \\
\text { study }\end{array}$ & $\begin{array}{l}1 \text { January to } \\
30 \text { March, } \\
2020\end{array}$ & $20 / 17$ & 37 & 17 & 20 & $62(59-70)$ & NLR & 6 \\
\hline $\begin{array}{l}\text { Ortiz-Brizuela } \\
\text { E et al. }{ }^{50}\end{array}$ & Mexico City & 2020 & $\begin{array}{l}\text { Tertiary Care Center located in } \\
\text { Mexico City }\end{array}$ & $\begin{array}{l}\text { Prospective } \\
\text { cohort study }\end{array}$ & $\begin{array}{l}26 \text { February } \\
\text { to } 11 \text { April, } \\
2020\end{array}$ & $85 / 55$ & $\begin{array}{l}140 \\
\text { (included } \\
\text { inpatients } \\
\text { only) }\end{array}$ & 111 & 29 & $\begin{array}{l}49.00 \\
(39.00-61.25)\end{array}$ & $\begin{array}{l}\text { CRP, PCT, ESR, } \\
\text { Ferritin }\end{array}$ & 7 \\
\hline Qin C et al. ${ }^{15}$ & China & 2020 & $\begin{array}{l}\text { Tongji } \\
\text { Hospital }\end{array}$ & $\begin{array}{l}\text { Retrospective } \\
\text { study }\end{array}$ & $\begin{array}{l}10 \text { January to } \\
12 \text { February, } \\
2020\end{array}$ & $235 / 217$ & 452 & 166 & 286 & $58(47-67)$ & $\begin{array}{l}\text { CRP, PCT, ESR, } \\
\text { NLR, Ferritin, } \\
\text { IL-6, IL-10, IL- } \\
2 R, \text { TNF- } \alpha\end{array}$ & 6 \\
\hline $\begin{array}{l}\text { Shahriarirad } \\
\text { R et al. } .^{51}\end{array}$ & Iran & 2020 & Shiraz University of Medical Sciences & $\begin{array}{l}\text { Retrospective } \\
\text { study }\end{array}$ & $\begin{array}{l}20 \text { February } \\
\text { to } 20 \text { March, } \\
2020\end{array}$ & $71 / 42$ & 113 & 102 & 11 & $53.75 \pm 16.58$ & CRP, ESR & 7 \\
\hline $\begin{array}{l}\text { Shang W } \\
\text { et al. }^{52}\end{array}$ & China & 2020 & Wuhan Forth Hospital & $\begin{array}{l}\text { Retrospective } \\
\text { study }\end{array}$ & $\begin{array}{l}16 \text { January to } \\
28 \text { February, } \\
2020\end{array}$ & $220 / 223$ & 443 & 304 & 139 & $\begin{array}{l}56.00 \\
(43.25-66.75)\end{array}$ & $\begin{array}{l}\text { CRP, PCT, ESR, } \\
\text { NLR }\end{array}$ & 7 \\
\hline Shao L et al. ${ }^{53}$ & China & 2020 & Zhongnan Hospital & $\begin{array}{l}\text { Retrospective } \\
\text { study }\end{array}$ & $\begin{array}{l}10 \text { January to } \\
8 \text { March, } \\
2020\end{array}$ & $62 / 93$ & 155 & 104 & 51 & $48(33-63)$ & CRP, IL-6 & 8 \\
\hline Shi J et al. ${ }^{54}$ & China & 2020 & $\begin{array}{l}\text { Four designated hospitals located in } \\
\text { Wenzhou, Wuhan, Huaihua, and } \\
\text { Shanghai }\end{array}$ & $\begin{array}{l}\text { Nested case- } \\
\text { control study }\end{array}$ & $\begin{array}{l}17 \text { January to } \\
1 \text { February, } \\
2020\end{array}$ & $49 / 36$ & 85 & 69 & 16 & $46.6 \pm 15.0$ & CRP & 8 \\
\hline
\end{tabular}


Table 1 (continued)

\begin{tabular}{|c|c|c|c|c|c|c|c|c|c|c|c|c|}
\hline $\begin{array}{l}\text { Spinetti T } \\
\text { et al. }^{55}\end{array}$ & Switzerland & 2020 & Inselspital, Bern University Hospital & $\begin{array}{l}\text { Prospective } \\
\text { study }\end{array}$ & $\begin{array}{l}\text { March to } \\
\text { April } 2020\end{array}$ & $12 / 4$ & 16 & 7 & 9 & $66(62-77)$ & CRP, PCT & 7 \\
\hline Sun $\mathrm{Y}$ et al. ${ }^{56}$ & China & 2020 & $\begin{array}{l}\text { The Fifth Medical Center of Chinese } \\
\text { PLA General Hospital }\end{array}$ & Cohort study & NA & $37 / 26$ & 63 & 44 & 19 & 47 (Range: 3-85) & CRP, ESR, IL-6 & 6 \\
\hline Tian $\mathrm{J}$ et al. ${ }^{57}$ & China & 2020 & $\begin{array}{l}\text { Tongji Hospital, Wuhan Union } \\
\text { Hospital, Wuhan First Hospital, the } \\
\text { Central Hospital of Wuhan, Wuhan } \\
\text { Fourth Hospital and Puai Hospital, } \\
\text { Fifth Hospital of Wuhan, Wuhan } \\
\text { Pulmonary Hospital, Wuhan } \\
\text { Jinyintan Hospital, and Wuhan } \\
\text { Hankou Hospital }\end{array}$ & $\begin{array}{l}\text { Retrospective } \\
\text { study }\end{array}$ & $\begin{array}{l}13 \text { January to } \\
18 \text { March, } \\
2020\end{array}$ & $119 / 113$ & $\begin{array}{l}232 \text { (taken } \\
\text { patients with } \\
\text { cancer only) }\end{array}$ & 84 & 148 & $64 \cdot 0(58 \cdot 0-69 \cdot 0)$ & $\begin{array}{l}\text { CRP, PCT, } \\
\text { Ferritin, IL-6, IL- } \\
\text { 10, IL-2R, TNF- } \alpha\end{array}$ & 8 \\
\hline Wan $S$ et al. ${ }^{58}$ & China & 2020 & $\begin{array}{l}\text { Chongqing Three Gorges Central } \\
\text { Hospital }\end{array}$ & $\begin{array}{l}\text { Retrospective } \\
\text { study }\end{array}$ & $\begin{array}{l}26 \text { January to } \\
4 \text { February } \\
2020\end{array}$ & $66 / 57$ & 123 & 102 & 21 & $\begin{array}{l}\text { Non-severe: } 43.05 \\
\pm 13.12 \\
\text { Severe: } 61.29 \pm \\
15.55\end{array}$ & $\begin{array}{l}\text { IL-6, IL-10, TNF- } \\
\alpha\end{array}$ & 6 \\
\hline $\begin{array}{l}\text { Wang G } \\
\text { et al. }^{59}\end{array}$ & China & 2020 & $\begin{array}{l}\text { Public Health Treatment } \\
\text { Center of Changsha }\end{array}$ & Case series & $\begin{array}{l}17 \text { January to } \\
20 \text { February, } \\
2020\end{array}$ & $105 / 104$ & 209 & 193 & 16 & $\begin{array}{l}\text { Non-severe: } 42 \\
\text { (Range:19-84); } \\
\text { Severe: } 54 \text { (Range: } \\
\text { 35-68) }\end{array}$ & CRP, ESR & 8 \\
\hline $\begin{array}{l}\text { Wang L } \\
\text { et al. }^{60}\end{array}$ & China & 2020 & $\begin{array}{l}\text { People's Hospital of Qiandongnan } \\
\text { Miao and Dong autonomous } \\
\text { prefecture \& Qiannan Miao and Buyi } \\
\text { autonomous prefecture }\end{array}$ & $\begin{array}{l}\text { Retrospective } \\
\text { study }\end{array}$ & $\begin{array}{l}23 \text { January to } \\
29 \text { February, } \\
2020\end{array}$ & $13 / 14$ & 27 & 23 & 4 & $33.23 \pm 13.21$ & CRP & 6 \\
\hline $\begin{array}{l}\text { Wang R } \\
\text { et al. }\end{array}$ & China & 2020 & $\begin{array}{l}\text { NO.2 People's Hospital of Fuyang } \\
\text { City }\end{array}$ & $\begin{array}{l}\text { Retrospective } \\
\text { study }\end{array}$ & $\begin{array}{l}20 \text { January to } \\
9 \text { February, } \\
2020\end{array}$ & $71 / 54$ & 125 & 100 & 25 & $38.76 \pm 13.799$ & $\begin{array}{l}\text { CRP, PCT, SAA, } \\
\text { IL-6 }\end{array}$ & 7 \\
\hline $\begin{array}{l}\text { Wang Y } \\
\text { et al. }^{62}\end{array}$ & China & 2020 & Guangzhou Eighth People's Hospital & $\begin{array}{l}\text { Retrospective } \\
\text { study }\end{array}$ & $\begin{array}{l}20 \text { January to } \\
10 \text { February, } \\
2020\end{array}$ & $128 / 147$ & 275 & 230 & 45 & $49(34-62)$ & CRP, PCT & 8 \\
\hline $\begin{array}{l}\text { Wang Z } \\
\text { et al. }^{63}\end{array}$ & China & 2020 & Union hospital & Cohort study & $\begin{array}{l}16 \text { January to } \\
29 \text { January, } \\
2020\end{array}$ & $32 / 37$ & 69 & 55 & 14 & $42.0(35.0-62.0)$ & $\begin{array}{l}\text { CRP, PCT, ESR, } \\
\text { IL-6, IL-10, TNF- } \\
\alpha\end{array}$ & 7 \\
\hline $\begin{array}{l}\text { Wei X et al. } \\
\text { (b) })^{64}\end{array}$ & China & 2020 & Union Hospital & $\begin{array}{l}\text { Retrospective } \\
\text { study }\end{array}$ & $\begin{array}{l}13 \text { February } \\
\text { to } 3 \text { March, } \\
2020\end{array}$ & $130 / 122$ & 252 & 131 & 121 & $64 \cdot 8 \pm 13 \cdot 3$ & $\begin{array}{l}\text { PCT, IL-10, TNF- } \\
\alpha\end{array}$ & 6 \\
\hline $\begin{array}{l}\text { Wei } X \text { et al. } \\
\text { (a) })^{65}\end{array}$ & China & 2020 & Union Hospital & $\begin{array}{l}\text { Retrospective } \\
\text { study }\end{array}$ & $\begin{array}{l}1 \text { February to } \\
3 \text { March, } \\
2020\end{array}$ & $305 / 292$ & 597 & 394 & 203 & $66(59-72)$ & CRP, IL-6 & 6 \\
\hline Wu C et al. ${ }^{66}$ & China & 2020 & Jinyintan Hospital & $\begin{array}{l}\text { Retrospective } \\
\text { study }\end{array}$ & $\begin{array}{l}25 \text { December, } \\
2019 \text { to } 26 \\
\text { January, } \\
2020\end{array}$ & $128 / 73$ & 201 & 117 & 84 & $51(43-60)$ & $\begin{array}{l}\text { ESR, IL-6, } \\
\text { Ferritin }\end{array}$ & 8 \\
\hline Xie $\mathrm{H}$ et al. ${ }^{67}$ & China & 2020 & Jinyintan Hospital. & $\begin{array}{l}\text { Retrospective } \\
\text { study }\end{array}$ & $\begin{array}{l}2 \text { February to } \\
23 \text { February, } \\
2020\end{array}$ & $44 / 35$ & 79 & 51 & 28 & $60.0(48.0-66.0)$ & ESR, PCT & 7 \\
\hline Xu B et al. ${ }^{68}$ & China & 2020 & $\begin{array}{l}\text { Hubei Provincial Hospital of } \\
\text { traditional Chinese and Western } \\
\text { medicine }\end{array}$ & $\begin{array}{l}\text { Retrospective } \\
\text { study }\end{array}$ & $\begin{array}{l}26 \text { December, } \\
2019 \text { to } 1 \\
\text { March, } 2020\end{array}$ & $103 / 84$ & 187 & 80 & 107 & $62(48.5-71)$ & $\begin{array}{l}\text { CRP, PCT, SAA, } \\
\text { IL-6, IL-10 }\end{array}$ & 8 \\
\hline $\begin{array}{l}\text { Yang A } \\
\text { et al. }^{69}\end{array}$ & China & 2020 & $\begin{array}{l}\text { Chongqing Public Health Medical } \\
\text { Treatment Center }\end{array}$ & $\begin{array}{l}\text { Retrospective } \\
\text { study }\end{array}$ & $\begin{array}{l}24 \text { January to } \\
7 \text { February, } \\
2020\end{array}$ & $56 / 58$ & 114 & 99 & 15 & $46.5 \pm 15.15$ & ESR & 6 \\
\hline $\begin{array}{l}\text { Yang AP } \\
\text { et al. }{ }^{70}\end{array}$ & China & 2020 & $\begin{array}{l}\text { Zhejiang Xiaoshan Hospital, } \\
\text { The First Affiliated Hospital of } \\
\text { Nanchang University }\end{array}$ & $\begin{array}{l}\text { Retrospective } \\
\text { study }\end{array}$ & $\begin{array}{l}\text { Until } 20 \\
\text { February, } \\
2020\end{array}$ & $56 / 37$ & 93 & 69 & 24 & $46.4 \pm 17.6$ & CRP, NLR & 8 \\
\hline Yang L et al. ${ }^{71}$ & China & 2020 & Yichang Central People's Hospital & $\begin{array}{l}\text { Descriptive } \\
\text { study }\end{array}$ & & $98 / 102$ & 200 & 171 & 29 & $55 \pm 17.1$ & CRP & 7 \\
\hline
\end{tabular}




\begin{tabular}{|c|c|c|c|c|c|c|c|c|c|c|c|c|}
\hline \multicolumn{13}{|c|}{2020} \\
\hline $\begin{array}{l}\text { Yang Q } \\
\text { et al. }^{72}\end{array}$ & China & 2020 & Wuhan Third Hospital & $\begin{array}{l}\text { Retrospective } \\
\text { study }\end{array}$ & $\begin{array}{l}28 \text { January to } \\
12 \text { February, } \\
2020\end{array}$ & $66 / 70$ & 136 & 103 & 33 & $56(44-64)$ & CRP & 6 \\
\hline Yao Q et al. ${ }^{73}$ & China & 2020 & Dabieshan Medical Center & $\begin{array}{l}\text { Retrospective } \\
\text { cohort study }\end{array}$ & $\begin{array}{l}30 \text { January to } \\
11 \text { February, } \\
2020\end{array}$ & $43 / 65$ & 108 & 83 & 25 & $52(37-58)$ & РCT & 8 \\
\hline $\begin{array}{l}\text { Young BE } \\
\text { et al. }^{74}\end{array}$ & Singapore & 2020 & 4 hospitals & $\begin{array}{l}\text { Descriptive } \\
\text { case series }\end{array}$ & $\begin{array}{l}23 \text { January to } \\
\text { 3 February, } \\
2020\end{array}$ & 9/9 & 18 & 12 & 6 & 47 (Range: $31-73$ ) & CRP & 7 \\
\hline $\begin{array}{l}\text { Zeng QL } \\
\text { et al. }^{75}\end{array}$ & China & 2020 & $\begin{array}{l}12 \text { hospitals in Henan and Shaanxi } \\
\text { Provinces }\end{array}$ & $\begin{array}{l}\text { Retrospective } \\
\text { study }\end{array}$ & $\begin{array}{l}20 \text { January to } \\
8 \text { February, } \\
2020\end{array}$ & $91 / 58$ & 149 & 122 & 27 & $42(30-55)$ & CRP, ESR, PCT & 7 \\
\hline $\begin{array}{l}\text { Zhang JJ } \\
\text { et al. }^{76}\end{array}$ & China & 2020 & No. 7 Hospital of Wuhan & $\begin{array}{l}\text { Retrospective } \\
\text { study }\end{array}$ & $\begin{array}{l}16 \text { January to } \\
3 \text { February, } \\
2020\end{array}$ & $71 / 69$ & 140 & 82 & 58 & 57 (Range: 25-87) & CRP, PCT, SAA & 6 \\
\hline $\begin{array}{l}\text { Zhang Y } \\
\text { et al. }{ }^{77}\end{array}$ & China & 2020 & Zhongnan Hospital & $\begin{array}{l}\text { Retrospective } \\
\text { study }\end{array}$ & $\begin{array}{l}18 \text { January to } \\
22 \text { February, } \\
2020\end{array}$ & $49 / 66$ & 115 & 84 & 31 & $49.52 \pm 17.06$ & NLR & 7 \\
\hline $\begin{array}{l}\text { Zheng } \mathrm{F} \\
\text { et al. }^{78}\end{array}$ & China & 2020 & $\begin{array}{l}\text { North Hospital of Changsha First } \\
\text { Hospital }\end{array}$ & $\begin{array}{l}\text { Retrospective } \\
\text { study }\end{array}$ & $\begin{array}{l}17 \text { January to } \\
7 \text { February, } \\
2020\end{array}$ & $80 / 81$ & 161 & 131 & 30 & $45(33.5-57)$ & CRP & 6 \\
\hline $\begin{array}{l}\text { Zheng Y } \\
\text { et al. }\end{array}$ & China & 2020 & $\begin{array}{l}\text { Chengdu } \\
\text { Public Health Clinical Medical } \\
\text { Center }\end{array}$ & $\begin{array}{l}\text { Retrospective } \\
\text { study }\end{array}$ & $\begin{array}{l}16 \text { January to } \\
20 \text { February, } \\
2020\end{array}$ & $51 / 48$ & 99 & 67 & 32 & $49.4 \pm 18.45$ & CRP & 7 \\
\hline Zhu Z et al. ${ }^{80}$ & China & 2020 & $\begin{array}{l}\text { Hwa Mei Hospital, University of } \\
\text { Chinese Academy of Sciences }\end{array}$ & $\begin{array}{l}\text { Retrospective } \\
\text { study }\end{array}$ & $\begin{array}{l}23 \text { January to } \\
20 \text { February, } \\
2020\end{array}$ & $45 / 82$ & 127 & 111 & 16 & $50.90 \pm 15.26$ & $\begin{array}{l}\text { CRP, ESR, NLR, } \\
\text { IL-6, IL-10, TNF- } \\
\alpha\end{array}$ & 7 \\
\hline \multicolumn{13}{|c|}{ Studies stratified by mortality } \\
\hline Author & Country & $\begin{array}{c}\begin{array}{c}\text { Year of } \\
\text { publication }\end{array} \\
\end{array}$ & Hospitals & $\begin{array}{c}\text { Type of } \\
\text { publication }\end{array}$ & $\begin{array}{c}\text { Date of data } \\
\text { collection }\end{array}$ & $\begin{array}{c}\text { Gender } \\
(\mathrm{M} / \mathrm{F})\end{array}$ & $\begin{array}{c}\text { Total } \\
\text { patients }\end{array}$ & Survivors & $\begin{array}{c}\text { Non- } \\
\text { survivors }\end{array}$ & $\begin{array}{l}\text { Age, median (IQR) } \\
\text { or mean (SD) }\end{array}$ & $\begin{array}{c}\text { Parameters } \\
\text { extracted }\end{array}$ & NOS \\
\hline $\begin{array}{l}\text { Bonetti G } \\
\text { et al. }^{81}\end{array}$ & Italy & 2020 & Valcamonica Hospital & $\begin{array}{l}\text { Retrospective } \\
\text { study }\end{array}$ & $\begin{array}{l}1 \text { March to } 30 \\
\text { March, } 2020\end{array}$ & $96 / 48$ & 144 & 74 & 70 & $\begin{array}{l}\text { Survivors: } 62.1 \\
\text { (53.0-72.8) } \\
\text { Non-survivors: } 78.0 \\
(64.2-84.0)\end{array}$ & CRP, Ferritin & 8 \\
\hline $\begin{array}{l}\text { Chen } R \\
\text { et al. }{ }^{14}\end{array}$ & China & 2020 & 575 hospitals throughout China & $\begin{array}{l}\text { Retrospective } \\
\text { study }\end{array}$ & $\begin{array}{l}\text { Till March 22, } \\
2020\end{array}$ & $313 / 235$ & 548 & 445 & 103 & $56.0 \pm 14.5$ & $\begin{array}{l}\text { CRP, PCT, NLR, } \\
\text { SAA, Ferritin, IL- } \\
6\end{array}$ & 7 \\
\hline Chen T et al. ${ }^{82}$ & China & 2020 & Tongji Hospital & $\begin{array}{l}\text { Retrospective } \\
\text { case series }\end{array}$ & $\begin{array}{l}13 \text { January to } \\
12 \text { February, } \\
2020\end{array}$ & $171 / 103$ & 274 & 161 & 113 & $62.0(44.0-70.0)$ & ESR, Ferritin & 7 \\
\hline $\begin{array}{l}\text { Chen TL } \\
\text { et al. }{ }^{.33}\end{array}$ & China & 2020 & Zhongnan Hospital & $\begin{array}{l}\text { Retrospective } \\
\text { study }\end{array}$ & $\begin{array}{l}1 \text { January to } \\
10 \text { February, } \\
2020\end{array}$ & $34 / 21$ & 55 & 36 & 19 & $74(65-91)$ & PCT & 8 \\
\hline $\begin{array}{l}\text { Covino M } \\
\text { et al. }^{84}\end{array}$ & Italy & 2020 & Urban Teaching Hospital & $\begin{array}{l}\text { Retrospective } \\
\text { study }\end{array}$ & $\begin{array}{l}1 \text { March to } 31 \\
\text { March, } 2020\end{array}$ & $37 / 32$ & 69 & 46 & 23 & $84(82-89)$ & $\begin{array}{l}\text { CRP, PCT, } \\
\text { Ferritin }\end{array}$ & 8 \\
\hline $\begin{array}{l}\text { Deng Y } \\
\text { et al. }\end{array}$ & China & 2020 & $\begin{array}{l}\text { Hankou and Caidian branch of } \\
\text { Tongji Hospital, and Hankou branch } \\
\text { of The Central Hospital of Wuhan }\end{array}$ & $\begin{array}{l}\text { Retrospective } \\
\text { study }\end{array}$ & $\begin{array}{l}1 \text { January to } \\
21 \text { February, } \\
2020\end{array}$ & $124 / 101$ & 225 & 116 & 109 & $\begin{array}{l}\text { Survivors: } 40 \\
\text { (33-57) } \\
\text { Non-survivors: } 69 \\
(62-74)\end{array}$ & CRP & 6 \\
\hline $\begin{array}{l}\text { Giacomelli A } \\
\text { et al. }^{86}\end{array}$ & Italy & 2020 & Luigi Sacco Hospital, Milan & $\begin{array}{l}\text { Prospective } \\
\text { study }\end{array}$ & $\begin{array}{l}21 \text { February } \\
\text { to } 19 \text { March, } \\
2020\end{array}$ & $161 / 72$ & 233 & 185 & 48 & $61(50-72)$ & CRP & 8 \\
\hline
\end{tabular}




\begin{tabular}{|c|c|c|c|c|c|c|c|c|c|c|c|c|}
\hline $\begin{array}{l}\text { Huang J } \\
\text { et al. }{ }^{87}\end{array}$ & China & 2020 & $\begin{array}{l}\text { Third People's Hospital of Yichang, } \\
\text { Hubei }\end{array}$ & $\begin{array}{l}\text { Retrospective } \\
\text { study }\end{array}$ & $\begin{array}{l}25 \text { January to } \\
24 \text { March, } \\
2020\end{array}$ & $160 / 139$ & 299 & 283 & 16 & $53.4 \pm 16.7$ & $\begin{array}{l}\text { CRP, PCT, ESR, } \\
\text { NLR }\end{array}$ & 8 \\
\hline $\begin{array}{l}\text { Javanian M } \\
\text { et al. }{ }^{88}\end{array}$ & Iran & 2020 & $\begin{array}{l}\text { Ayatollah Rohani, Shahid Beheshti } \\
\text { and Yahyanejad hospitals }\end{array}$ & $\begin{array}{l}\text { Retrospective } \\
\text { study }\end{array}$ & $\begin{array}{l}25 \text { February } \\
\text { to } 12 \text { March, } \\
2020\end{array}$ & $51 / 49$ & 100 & 81 & 19 & $60.12 \pm 13.87$ & CRP & 8 \\
\hline Lee JY et al. ${ }^{89}$ & Korea & 2020 & 4 Hospitals & $\begin{array}{l}\text { Retrospective } \\
\text { study }\end{array}$ & $\begin{array}{l}18 \text { February } \\
\text { to } 4 \text { March, } \\
2020\end{array}$ & $44 / 54$ & 98 & 78 & 20 & $72(68-79)$ & CRP & 8 \\
\hline Li J et al. ${ }^{44}$ & China & 2020 & Central Hospital of Wuhan & $\begin{array}{l}\text { Retrospective } \\
\text { study }\end{array}$ & $\begin{array}{l}1 \text { January to } \\
20 \text { February, } \\
2020\end{array}$ & $36 / 23$ & $\begin{array}{l}59 \text { (included } \\
\text { critical } \\
\text { patients } \\
\text { only) }\end{array}$ & 17 & 42 & $\begin{array}{l}67.00 \\
(56.00-75.00)\end{array}$ & PCT, CRP & 7 \\
\hline Li L et al. ${ }^{90}$ & China & 2020 & Union Hospital & $\begin{array}{l}\text { Retrospective } \\
\text { study }\end{array}$ & $\begin{array}{l}10 \text { January to } \\
22 \text { February, } \\
2020\end{array}$ & $41 / 52$ & 93 & 68 & 25 & $51.0 \pm 17.5$ & $\begin{array}{l}\text { ESR, NLR, } \\
\text { Ferritin }\end{array}$ & 8 \\
\hline Luo $\mathrm{X}$ et al. ${ }^{91}$ & China & 2020 & $\begin{array}{l}\text { Eastern Campus of Renmin Hospital } \\
\text { of Wuhan University }\end{array}$ & $\begin{array}{l}\text { Retrospective } \\
\text { study }\end{array}$ & $\begin{array}{l}30 \text { January to } \\
20 \text { February, } \\
2020\end{array}$ & $150 / 148$ & 298 & 214 & 84 & $57(40-69)$ & CRP, PCT, NLR & 8 \\
\hline $\begin{array}{l}\text { Masetti C } \\
\text { et al. }^{92}\end{array}$ & Italy & 2020 & IRCCS, Rozzano & $\begin{array}{l}\text { Retrospective } \\
\text { study }\end{array}$ & $\begin{array}{l}28 \text { February } \\
\text { to } 10 \text { April, } \\
2020\end{array}$ & $148 / 81$ & 229 & 196 & 33 & $60.7 \pm 14.2$ & CRP, Ferritin & 8 \\
\hline Pan $\mathrm{F}$ et al..$^{93}$ & China & 2020 & Union Hospital & $\begin{array}{l}\text { Case-control } \\
\text { study }\end{array}$ & $\begin{array}{l}27 \text { January to } \\
19 \text { March, } \\
2020\end{array}$ & $85 / 39$ & 124 & 35 & 89 & $68(61-75)$ & CRP, PCT & 8 \\
\hline $\begin{array}{l}\text { Ruan } Q \\
\text { et al. }{ }^{94}\end{array}$ & China & 2020 & $\begin{array}{l}\text { Jin Yin-tan Hospital and Tongji } \\
\text { Hospital }\end{array}$ & $\begin{array}{l}\text { Retrospective } \\
\text { study }\end{array}$ & NA & $102 / 48$ & 150 & 82 & 68 & $\begin{array}{l}\text { Survivors: } 50 \\
\text { (44-81) } \\
\text { Non-survivors: } 67 \\
(15-81)\end{array}$ & $\begin{array}{l}\text { CRP, Ferritin, IL- } \\
6\end{array}$ & 7 \\
\hline $\begin{array}{l}\text { Satici C } \\
\text { et al. } .95\end{array}$ & Turkey & 2020 & $\begin{array}{l}\text { Gaziosmanpasa Research and } \\
\text { Training Hospital, University of } \\
\text { Health Sciences }\end{array}$ & $\begin{array}{l}\text { Retrospective } \\
\text { study }\end{array}$ & $\begin{array}{l}2 \text { April to } 1 \\
\text { May, } 2020\end{array}$ & $347 / 334$ & 681 & 626 & 55 & $56.9 \pm 15.7$ & CRP, Ferritin & 8 \\
\hline $\begin{array}{l}\text { Shahriarirad } \\
\mathrm{R} \text { et al. }{ }^{51}\end{array}$ & Iran & 2020 & $\begin{array}{l}\text { Centers for COVID-19 diagnosis and } \\
\text { under the management of Shiraz } \\
\text { University of Medical Sciences }\end{array}$ & $\begin{array}{l}\text { Retrospective } \\
\text { study }\end{array}$ & $\begin{array}{l}20 \text { February } \\
\text { to } 20 \text { March, } \\
2020\end{array}$ & $71 / 42$ & 113 & 104 & 9 & $53.75 \pm 16.58$ & CRP, ESR & 7 \\
\hline Shi $S$ et al..$^{96}$ & China & 2020 & $\begin{array}{l}\text { Renmin Hospital of Wuhan } \\
\text { University }\end{array}$ & $\begin{array}{l}\text { Retrospective } \\
\text { study }\end{array}$ & $\begin{array}{l}1 \text { January to } \\
23 \text { February, } \\
2020\end{array}$ & $322 / 349$ & 671 & 609 & 62 & $63(50-72)$ & CRP, PCT & 8 \\
\hline Sun $\mathrm{H}$ et al. ${ }^{97}$ & China & 2020 & $\begin{array}{l}\text { Sino-French New City Branch of } \\
\text { Tongji Hospital }\end{array}$ & $\begin{array}{l}\text { Retrospective } \\
\text { case-control. }\end{array}$ & $\begin{array}{l}29 \text { January to } \\
5 \text { March, } \\
2020\end{array}$ & $133 / 111$ & 244 & 123 & 121 & $\begin{array}{l}\text { Survivors: } 67 \\
\text { (64-72); Non- } \\
\text { survivors: } 72 \\
(66-78)\end{array}$ & $\begin{array}{l}\text { ESR, PCT, } \\
\text { Ferritin, IL-6 }\end{array}$ & 8 \\
\hline $\begin{array}{l}\text { Tomlins } J \\
\text { et al. }^{98}\end{array}$ & UK & 2020 & North Bristol NHS Trust & $\begin{array}{l}\text { Retrospective } \\
\text { study }\end{array}$ & $\begin{array}{l}10 \text { March to } \\
30 \text { March, } \\
2020\end{array}$ & $60 / 35$ & 95 & 75 & 20 & $75(59-82)$ & $\begin{array}{l}\text { CRP, NLR, } \\
\text { Ferritin }\end{array}$ & 7 \\
\hline Tu WJ et al..$^{99}$ & China & 2020 & Zhongnan Hospital & $\begin{array}{l}\text { Retrospective } \\
\text { study }\end{array}$ & $\begin{array}{l}3 \text { January to } \\
24 \text { February, } \\
2020\end{array}$ & $79 / 95$ & 174 & 149 & 25 & $\begin{array}{l}\text { Survivors: } 51 \\
\text { (37-62); Non- } \\
\text { survivors: } 70 \\
(64-80)\end{array}$ & CRP, IL-6 & 8 \\
\hline $\begin{array}{l}\text { Wang } \mathrm{L} \\
\text { et al. }^{100}\end{array}$ & China & 2020 & $\begin{array}{l}\text { Renmin Hospital of Wuhan } \\
\text { University }\end{array}$ & $\begin{array}{l}\text { Retrospective } \\
\text { study }\end{array}$ & $\begin{array}{l}1 \text { January to } 6 \\
\text { February, } \\
2020\end{array}$ & $166 / 173$ & 339 & 274 & 65 & $69(65-76)$ & IL-6 & 8 \\
\hline $\begin{array}{l}\text { Wang Y } \\
\text { et al. }{ }^{17}\end{array}$ & China & 2020 & Tongji hospital & Case series & $\begin{array}{l}25 \text { January to } \\
25 \text { February, } \\
2020\end{array}$ & $179 / 165$ & 344 & 211 & 133 & $64(52-72)$ & $\begin{array}{l}\text { PCT, IL-6, IL-10, } \\
\text { IL-2R, TNF- } \alpha\end{array}$ & 8 \\
\hline Wu C et al. ${ }^{66}$ & China & 2020 & Jinyintan Hospital & $\begin{array}{l}\text { Retrospective } \\
\text { study }\end{array}$ & $\begin{array}{l}25 \text { December, } \\
2019 \text { to } 26\end{array}$ & $60 / 24$ & & 40 & 44 & $58.5(50.0-69.0)$ & ESR & 8 \\
\hline
\end{tabular}


Table 1 (continued)

\begin{tabular}{|c|c|c|c|c|c|c|c|c|c|c|c|c|}
\hline & & & & & $\begin{array}{l}\text { January, } \\
2020\end{array}$ & & $\begin{array}{l}84 \text { (included } \\
\text { patients with } \\
\text { ARDS only) }\end{array}$ & & & & & \\
\hline Yan X et al. ${ }^{101}$ & China & 2020 & $\begin{array}{l}\text { Wuhan Third Hospital \& Tongren } \\
\text { Hospital of Wuhan University }\end{array}$ & $\begin{array}{l}\text { Retrospective } \\
\text { study }\end{array}$ & $\begin{array}{l}11 \text { January to } \\
3 \text { March, } \\
2020\end{array}$ & $493 / 511$ & 1004 & 964 & 40 & $\begin{array}{l}\text { Survivors: } 62 \\
\text { (50-70); Non- } \\
\text { survivors: } 68 \\
\text { (58-79) }\end{array}$ & NLR & 8 \\
\hline $\begin{array}{l}\text { Yang K } \\
\text { et al. }{ }^{102}\end{array}$ & China & 2020 & $\begin{array}{l}\text { Cancer Center of Wuhan Union } \\
\text { Hospital, West Branch of Wuhan } \\
\text { Union } \\
\text { Hospital, Jin Yin-tan Hospital, } \\
\text { Wuhan Red Cross } \\
\text { Hospital, the Central Hospital of } \\
\text { Wuhan, Huanggang } \\
\text { Central Hospital, the First People's } \\
\text { Hospital Affiliated to Yangtze } \\
\text { University, Xianning Central } \\
\text { Hospital, and Suizhou Central } \\
\text { Hospital }\end{array}$ & $\begin{array}{l}\text { Retrospective } \\
\text { study }\end{array}$ & $\begin{array}{l}13 \text { January to } \\
18 \text { March, } \\
2020\end{array}$ & $96 / 109$ & 205 & 165 & 40 & $63(56-70)$ & PCT, IL-6 & 8 \\
\hline $\begin{array}{l}\text { Zhang } \mathrm{N} \\
\text { et al. }{ }^{103}\end{array}$ & China & 2020 & $\begin{array}{l}\text { WuGang General } \\
\text { Hospital (Wuhan, Hubei Province) } \\
\text { and The First Affiliated Hospital of } \\
\text { Hunan } \\
\text { University of Medicine (Hunan } \\
\text { Province) }\end{array}$ & $\begin{array}{l}\text { Retrospective } \\
\text { study }\end{array}$ & $\begin{array}{l}9 \text { January to } \\
19 \text { February, } \\
2020\end{array}$ & $43 / 17$ & 60 & 50 & 10 & $64.4 \pm 11.0$ & CRP, NLR & 7 \\
\hline $\begin{array}{l}\text { Zhou F } \\
\text { et al. }{ }^{104}\end{array}$ & China & 2020 & $\begin{array}{l}135 \text { from Jinyintan Hospital and } 56 \\
\text { from Wuhan Pulmonary Hospital }\end{array}$ & $\begin{array}{l}\text { Retrospective } \\
\text { study }\end{array}$ & $\begin{array}{l}29 \text { December, } \\
2019 \text { to } 31 \\
\text { January, } \\
2020\end{array}$ & $119 / 72$ & 191 & 137 & 54 & $56.0(46.0-67.0)$ & $\begin{array}{l}\text { PCT, Ferritin, IL- } \\
6\end{array}$ & 6 \\
\hline
\end{tabular}

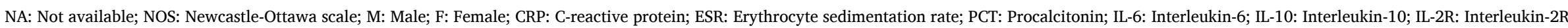
TNF- $\alpha$ : Tumor necrosis factor- $\alpha$; SAA: Serum amyloid A; NLR: Neutrophil to lymphocyte ratio. 
Table 2

Association of inflammatory parameters with disease severity in patients of COVID-19.

\begin{tabular}{|c|c|c|c|c|c|c|c|c|}
\hline \multirow[t]{2}{*}{ Inflammatory parameters } & \multirow[t]{2}{*}{ Number of studies } & \multirow[t]{2}{*}{ Participants } & \multicolumn{4}{|l|}{ Statistical method } & \multicolumn{2}{|c|}{ Heterogeneity } \\
\hline & & & SDM $(95 \% \mathrm{CI})$ & $\mathrm{MD}(95 \% \mathrm{CI})$ & Model & p-value & $\mathrm{I}^{2}$ & $\mathrm{P}_{\mathrm{h}}$-value \\
\hline CRP & 44 & 7898 & $1.14(0.97,1.32)$ & - & REM & $<0.00001$ & $90 \%$ & $<0.00001$ \\
\hline ESR & 17 & 3437 & - & $12.08(8.04,16.11)$ & REM & $<0.00001$ & $75 \%$ & $<0.00001$ \\
\hline PCT & 30 & 5899 & $0.88(0.68,1.08)$ & - & REM & $<0.00001$ & $90 \%$ & $<0.00001$ \\
\hline IL-6 & 18 & 3618 & - & $16.94(12.72,21.16)$ & REM & $<0.00001$ & $96 \%$ & $<0.00001$ \\
\hline IL-10 & 8 & 1626 & - & $2.03(1.36,2.70)$ & REM & $<0.00001$ & $82 \%$ & $<0.00001$ \\
\hline IL-2R & 2 & 531 & - & $238.26(31.90,444.62)$ & REM & 0.02 & $84 \%$ & 0.01 \\
\hline TNF- $\alpha$ & 7 & 1440 & - & $0.05(-0.57,0.68)$ & REM & 0.87 & $91 \%$ & $<0.00001$ \\
\hline Ferritin & 9 & 1609 & $0.71(0.60,0.81)$ & - & FEM & $<0.00001$ & $5 \%$ & 0.39 \\
\hline SAA & 8 & 1289 & $1.16(0.64,1.68)$ & - & REM & $<0.0001$ & $93 \%$ & $<0.00001$ \\
\hline NLR & 12 & 2519 & - & $3.27(1.99,4.55)$ & REM & $<0.00001$ & $90 \%$ & $<0.00001$ \\
\hline
\end{tabular}

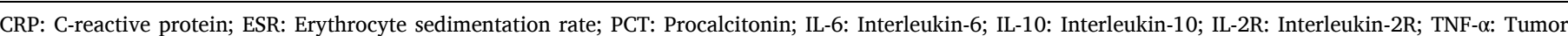

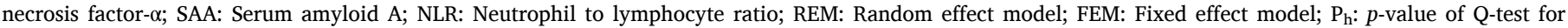
heterogeneity.

Table 3

Association of inflammatory parameters with mortality in COVID-19 patients.

\begin{tabular}{|c|c|c|c|c|c|c|c|c|}
\hline \multirow[t]{2}{*}{ Inflammatory parameters } & \multirow[t]{2}{*}{ Number of studies } & \multirow[t]{2}{*}{ Participants } & \multicolumn{4}{|l|}{ Statistical method } & \multicolumn{2}{|c|}{ Heterogeneity } \\
\hline & & & SDM $(95 \% \mathrm{CI})$ & $\mathrm{MD}(95 \% \mathrm{CI})$ & Model & $\mathrm{p}$-value & $\mathrm{I}^{2}$ & $\mathrm{P}_{\mathrm{h}}$-value \\
\hline CRP & 19 & 4318 & $1.18(0.80,1.55)$ & - & REM & $<0.00001$ & $94 \%$ & $<0.00001$ \\
\hline ESR & 7 & 1162 & - & $12.98(-1.79,27.75)$ & REM & 0.08 & $91 \%$ & $<0.00001$ \\
\hline PCT & 12 & 3022 & - & $0.26(0.18,0.34)$ & REM & $<0.00001$ & $85 \%$ & $<0.00001$ \\
\hline IL-6 & 8 & 2065 & - & $15.62(10.67,20.57)$ & REM & $<0.00001$ & $96 \%$ & $<0.00001$ \\
\hline Ferritin & 11 & 2554 & $0.95(0.74,1.17)$ & - & REM & $<0.00001$ & $76 \%$ & $<0.0001$ \\
\hline NLR & 7 & 2350 & - & $8.96(3.97,13.95)$ & REM & 0.0004 & $99 \%$ & $<0.00001$ \\
\hline
\end{tabular}

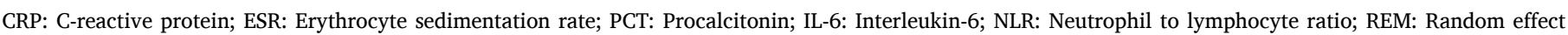
model; $\mathrm{P}_{\mathrm{h}}$ : $p$-value of $\mathrm{Q}$-test for heterogeneity.

In mortality studies, a funnel plot regarding the CRP showed that the $p$ value of Egger's test was 0.1058, suggesting no stable evidence of publication bias. Regression-based Egger's test showed statistically significant small-study effects for PCT $(p=0.0005)$ and ferritin $(p=$ 0.0449). The trim and fill method imputed 2 studies in the PCT whereas no study was imputed in ferritin (Supplement 7 and supplement 8).

\subsection{Sensitivity analysis}

Sensitivity analysis indicated that the combined results did not change with the exclusion of any one of the studies in CRP, ESR, PCT, IL6 , IL-10, TNF- $\alpha$, ferritin, SAA, and NLR between the severe and nonsevere groups (Supplement 9). Similarly, sensitivity analysis revealed that the results were not influenced with the exclusion of any one of the studies in CRP, ESR, PCT, ferritin, and NLR between the survivor and non-survivor groups. However, for IL-6, the pooled effect sizes changed after omitting Chen $\mathrm{R}$ et al. ${ }^{14}$ [MD $=24.48,95 \% \mathrm{CI}$ : $\left.17.25-31.71\right]$, Sun $\mathrm{H}$ et al. ${ }^{97}[\mathrm{MD}=10.31,95 \% \mathrm{CI}: 6.03-14.58]$, Wang Y et al. ${ }^{17}[\mathrm{MD}=$ 9.66, 95\% CI: 5.40-13.91], Yang K et al. ${ }^{102}[\mathrm{MD}=22.48$, 95\% CI: 15.84-29.12] and Zhou F et al. ${ }^{104}$ [MD $=22.33$, 95\% CI: $\left.15.65-29.01\right]$, separately (Supplement 10).

\section{Discussion}

This systematic review and metaanalysis included 83 studies to investigate the association of inflammatory parameters with the severity and mortality in COVID-19 patients. The findings revealed significantly higher levels of CRP, ESR, PCT, IL-6, IL-10, IL-2R, ferritin, SAA, and NLR in the severe group compared to the non-severe group with COVID-19. However, no significant difference was observed in the level of TNF- $\alpha$ between severe and non-severe groups. Similarly, the levels of CRP, PCT, IL-6, ferritin, and NLR were significantly higher in non-survivors compared with survivors whereas no significant difference was observed for ESR between survivors and non-survivors.
C-reactive protein is an acute-phase inflammatory protein produced by the liver and regulated at the transcriptional level by the cytokine IL6 and IL-1. ${ }^{105}$ It is an important index for diagnosing and evaluating severe pulmonary infectious diseases. ${ }^{106}$ SARS-CoV-2 shares similar clinical features with Middle East respiratory syndrome coronavirus ${ }^{107}$ and in patients with severe Middle East respiratory syndrome coronavirus pneumonia, increasing in C-reactive protein levels is correlated with clinical deterioration. ${ }^{108}$ Similarly, in our meta-analysis, elevated CRP was associated with both severity and mortality in COVID-19 patients, which represent more prominent inflammation in severe patients. ESR is a non-specific inflammatory marker, primarily reflecting changes in plasma protein types. ${ }^{109}$ In the present meta-analysis, a higher ESR level was associated with the severity of COVID-19. Similarly, the findings of systematic literature search and pooled analysis conducted by Lapic et al. ${ }^{110}$ stated that severe COVID-19 cases are associated with prominent elevations of ESR, as compared to non-severe cases. This increased ESR level in severe COVID-19 cases reflects a more profound inflammatory response and expression of acute-phase proteins. ${ }^{111}$

Procalcitonin, a peptide precursor of the hormone calcitonin, is normally synthesized and released by thyroid parafollicular C cells and is widely researched as a promising biomarker for the initial investigation of bacterial infection. ${ }^{112,113}$ Elevated PCT often occurs in sepsis and septic shock patients. ${ }^{114}$ In the present meta-analysis, an increased level of PCT was found to be associated with severity and mortality in COVID-19 patients. Similarly, Lippi et al. ${ }^{115}$ in their meta-analysis also demonstrated that increased PCT levels are associated with a 5-fold higher risk of severe COVID-19. During bacterial infection, the production and release of procalcitonin into the circulation from extrathyroidal sources is greatly amplified, which is maintained by increased levels of IL-6, IL-1 $\beta$, and TNF- $\alpha$ whereas the increased concentration of interferon- $\gamma$ during viral infection is negatively impacting the synthesis of PCT. ${ }^{112,116}$ This is why the level of PCT remains within the normal range in the majority of the patients with non-severe COVID-19 and increased value in severe COVID-19 may indicate secondary bacterial 
infection. $^{115}$

Elevated proinflammatory cytokine or chemokine responses induced immunopathology, described as a cytokine storm, has been involved in the pathogenesis of human coronavirus. ${ }^{13}$ It is hypothesized that SARS-CoV-2 first binds to alveolar epithelial cells and then the virus triggers the innate immune system and the adaptive immune system, leading to the release of a substantial number of cytokines, including IL-6, which is a pleiotropic cytokine important in regulating immunological and inflammatory responses. Abnormally increased levels of such cytokines or chemokines can cause tissue damage, resulting in respiratory failure or multiple organ failure. ${ }^{14,117-119}$ In addition to its strong proinflammatory function, IL-6 induces various acute-phase proteins, such as CRP, SAA, fibrinogen, antitrypsin, hepcidin, and components of complement to deteriorate inflammatory reactions and activate coagulation pathway with resultant disruption of procoagulant-anticoagulant homeostasis, induction of disseminated intravascular coagulation, and multi-organ failure. ${ }^{120,121}$ Among various cytokines and chemokines (IL-2, IL-8, IL-17, GCSF, IP-10, and TNF- $\alpha$ ) recognized, IL-6 has been considered as the most significant cytokines, which was found increased in both SARS and MERS, as well as in COVID-19. ${ }^{122-126}$ In our metaanalysis, high IL-6 has been linked with both severity and mortality in COVID-19 patients. The importance of identifying this elevated biomarker also lies in the potential use of an antibody against IL-6 such as tocilizumab, which has been reported to effectively improve clinical symptoms and repress the deterioration of severe and critical patients. ${ }^{127}$ Though IL-10 is an anti-inflammatory cytokine, a higher level of IL-10 was observed in the severe group compared to the non-severe group. Furthermore, higher IL-10 was also associated with mortality in COVID-19. ${ }^{17}$ This increased level of IL-10 may be because of the compensatory anti-inflammatory response. ${ }^{111}$ The present metaanalysis revealed association of higher level of IL-2R with COVID-19 severity. Wang et al. ${ }^{17}$ reported a higher level of IL-2R in non-survivors compared to survivors. Highly expressed IL-2R initiates autoreactive cytotoxic $\mathrm{CD}^{+}{ }^{+} \mathrm{T}$-cell-mediated autoimmunity. In the meantime, IL-2 promotes the proliferation of natural killer cells that strongly express IL-2R, facilitating the release of cytokines, which further induces the deadly "cytokine storm". ${ }^{128}$ Another potential proinflammatory biomarker for COVID-19 is TNF- $\alpha$, which facilitates the apoptosis of both lung epithelial cells and endothelial cells, leading to vascular leakage, alveolar edema, and hypoxia. ${ }^{13}$ It also mediates airway hyper-responsiveness and pathogenesis in influenza and SARS-CoV infection. ${ }^{129}$ In our meta-analysis, no significant difference was observed in the level of TNF- $\alpha$ between severe and non-severe COVID-19 groups. The possible reason for this insignificant difference could be due to insignificant difference in the level of TNF- $\alpha$ between severe and non-severe groups in 4 studies $^{58,63,64,80}$ and a significant decrease in the level of TNF- $\alpha$ in the severe group compared to the non-severe group in 1 study. ${ }^{16}$ However, 2 studies ${ }^{15,57}$ revealed a significant increase in TNF- $\alpha$ in the severe group compared to the non-severe group.

Serum ferritin is an acute-phase protein, which can be used as a prognostic marker for tissue damage or acute infections. ${ }^{130}$ Patients with COVID-19 in the severe group had a higher level of serum ferritin than those in the non-severe group. Furthermore, in our metaanalysis, a higher serum ferritin level was associated with mortality in COVID-19 patients. Though the pathophysiological background responsible for the association of hyperferritinemia and disease severity in patients with COVID-19 is not clearly grasped, it is suggested that hyperferritinemia in COVID-19 patients is most likely due to the cytokine storm and a secondary hemophagocytic lymphohistiocytosis. ${ }^{131}$ Serum amyloid A is a non-specific acute phase protein primarily produced in hepatocytes by cytokines IL- $1 \beta$, IL- 6 , and TNF- $\alpha$, and can be used as a prognostic marker for tissue injury or acute infections. ${ }^{132-135}$ It can promote inflammatory responses even at very low concentrations by activating chemokines and inducing chemotaxis. ${ }^{136,137}$ The level of SAA was found to be positively associated with the degree of pneumonia in SARS. ${ }^{138}$ Similarly, compared to the non-severe group, a significantly higher SAA level was observed in the severe group. Serum amyloid A was also found to be associated with mortality in COVID-19 and the increased SAA in non-survivors indicated the progressive immune-mediated damage in dead patients. ${ }^{14}$ Studies reported that severe/critical COVID-19 patients had large amounts of IL- $1 \beta$, IFN- $\gamma$, IP-10, MCP-1, MIP-1, TNF- $\alpha$, and other cytokines present in the system, which boost liver cells to produce SAA. ${ }^{39,139}$

Neutrophil-to-lymphocyte ratio (NLR) is the most well established inflammatory marker that reflects systemic inflammatory response and is easily obtainable through routine blood count analysis. ${ }^{140}$ Several COVID-19 patients had increased neutrophil counts and decreased lymphocyte counts during the severe phase of COVID-19 infection. ${ }^{141}$ Recently, the meta-analysis conducted by Lagunas-Rangel ${ }^{142}$ showed that the NLR values were significantly associated with the severity of COVID-19. Similarly, in our meta-analysis, elevated NLR was associated with severity and mortality in COVID-19 patients. This increased NLR reflects the enhanced inflammatory process in severe/critical COVID-19 patients. Therefore, the detection of NLR levels in COVID-19 patients may help in assessing disease severity.

This systematic review and meta-analysis had several limitations that should be addressed. First, we have excluded articles published in foreign languages and the articles in which the data were not presented as mean (standard deviation, SD) or median (interquartile range, IQR, or range), which may have introduced bias in the results. Second, we have converted non-normally distributed data to normally distributed data, which may have biased the results. Third, the majority of included studies were from China, which limits the generalizability of the results. Fourth, most of the included studies were retrospective and observational; therefore, the results obtained must be interpreted with caution. Lastly, substantial heterogeneity exists in almost all meta-analyses.

\section{Conclusion}

In conclusion, our systematic review and meta-analysis showed significant increased serum concentrations of CRP, ESR, PCT, IL-6, IL-10, IL-2R, ferritin, SAA, and NLR in severe COVID-19 patients when compared to those with non-severe COVID-19 patients. Similarly, we found significant increased levels of CRP, PCT, IL-6, ferritin, and NLR in non-survivors as compared to survivors. These inflammatory parameters could help the physicians to rapidly identify severe COVID-19 patients, hence facilitating the early initiation of effective treatment. In addition, these inflammatory parameters could be used to predict the transition from mild to severe/critical infection in patients of COVID-19.

\section{Authorship statement}

R.K. Mahat contributed to the concept, design, methodology, analysis, interpretation, supervision, writing, reviewing and editing. S. Panda contributed to the methodology, interpretation, writing, reviewing and editing. V. Rathore contributed to the methodology, analysis, interpretation, writing, reviewing and editing. S. Swain contributed to the methodology, supervision, reviewing and editing. L. Yadav contributed to the methodology, reviewing and editing. S.P. Sah contributed to the methodology, reviewing and editing.

\section{Appendix A. Supplementary data}

Supplementary data to this article can be found online at https://doi. org/10.1016/j.cegh.2021.100727.

\section{References}

1 Zhou P, Yang XL, Wang XG, et al. A pneumonia outbreak associated with a new coronavirus of probable bat origin. Nature. 2020;579(7798):270-273. https://doi. org/10.1038/s41586-020-2012-7. 
2 Zhu N, Zhang D, Wang W, et al. A novel coronavirus from patients with pneumonia in China, 2019. N Engl J Med. 2020;382(8):727-733. https://doi.org/10.1056/ NEJMoa2001017.

3 Statement on the second meeting of the International Health Regulations (2005) Emergency Committee regarding the outbreak of novel coronavirus (2019-nCoV). https://www.who.int/news-room/detail/30-01-2020-statement-on-th e-second-meeting-of-the-international-health -regulations-(2005)-emergency-committee-regarding-th e-outbreak-of-novel-coronavirus-(2019-ncov).

4 WHO. Coronavirus disease (COVID-2019) Situation Reports. World Health Organization; 2020. https://www.who.int/emergencies/diseases/novel-coron avirus-2019/situation-reports.

5 Chen N, Zhou M, Dong X, et al. Epidemiological and Clinical Characteristics of 99 Cases of 2019 Novel Coronavirus Pneumonia in Wuhan, China: A Descriptive Study. vol. 395. Lancet Elsevier Ltd; 2020:507-513.

6 Yang X, Yu Y, Xu J, et al. Clinical course and outcomes of critically ill patients with SARS-CoV-2 pneumonia in Wuhan, China: a single-centered, retrospective, observational study. Lancet Respir Med. 2020;8(5):475-481. https://doi.org/ 10.1016/S2213-2600(20)30079-5.

7 Wu Z, McGoogan JM. Characteristics of and important lessons from the coronavirus disease 2019 (COVID-19) outbreak in China: summary of a report of 72314 cases from the Chinese center for disease control and prevention. J Am Med Assoc. 2020; 323(13):1239-1242. https://doi.org/10.1001/jama.2020.2648.

8 Velavan TP, Meyer CG. Mild versus severe COVID-19: laboratory markers. Int $J$ Infect Dis. 2020;95:304-307. https://doi.org/10.1016/j.ijid.2020.04.061.

9 Weiss P, Murdoch DR. Clinical course and mortality risk of severe COVID-19. Lancet. 2020;395(10229):1014-1015. https://doi.org/10.1016/S0140-6736(20) 30633-4.

10 Cummings MJ, Baldwin MR, Abrams D, et al. Epidemiology, clinical course, and outcomes of critically ill adults with COVID-19 in New York City: a prospective cohort study. Lancet. 2020;395(10239):1763-1770. https://doi.org/10.1016/ S0140-6736(20)31189-2.

11 Henderson LA, Canna SW, Schulert GS, et al. On the alert for cytokine storm: immunopathology in COVID-19. Arthritis Rheum. 2020. https://doi.org/10.1002/ art.41285 [Published online on May 10].

12 Moore JB, June CH. Cytokine release syndrome in severe COVID-19. Science. 2020; 368(6490):473-474. https://doi.org/10.1126/science.abb8925.

13 Channappanavar R, Perlman S. Pathogenic human coronavirus infections: causes and consequences of cytokine storm and immunopathology. Semin Immunopathol. 2017;39(5):529-539. https://doi.org/10.1007/s00281-017-0629-x.

14 Chen R, Sang L, Jiang M, et al. Longitudinal hematologic and immunologic variations associated with the progression of COVID-19 patients in China. J Allergy Clin Immunol. 2020. https://doi.org/10.1016/j.jaci.2020.05.003 [published online on May 11].

15 Qin C, Zhou L, Hu Z, et al. Dysregulation of immune response in patients with coronavirus 2019 (COVID-19) in Wuhan, China. Clin Infect Dis. 2020;71(15): 762-768. https://doi.org/10.1093/cid/ciaa248.

$16 \mathrm{Lv}$ Z, Cheng S, Le J, et al. Clinical characteristics and co-infections of 354 hospitalized patients with COVID-19 in Wuhan, China: a retrospective cohort study. Microb Infect. 2020;22(4-5):195-199. https://doi.org/10.1016/j. micinf.2020.05.007.

17 Wang Y, Lu X, Li Y, et al. Clinical course and outcomes of 344 intensive care patients with COVID-19. Am J Respir Crit Care Med. 2020 Jun 1;201(11): 1430-1434. https://doi.org/10.1164/rccm.202003-0736LE.

18 Zeng F, Huang Y, Guo Y, et al. Association of inflammatory markers with the severity of COVID-19: a meta-analysis. Int J Infect Dis. 2020;96:467-474. https:// doi.org/10.1016/j.ijid.2020.05.055 [Published online on May 18] doi:.

19 Feng X, Li S, Sun Q, et al. Immune-inflammatory parameters in COVID-19 cases: a systematic review and meta-analysis. Front Med. 2020;7:301. https://doi.org/ 10.3389/fmed.2020.00301. Published 2020 Jun 9.

20 Moher D, Liberati A, Tetzlaff J, Altman DG. The PRISMA group. Preferred reporting Items for systematic reviews and meta-analyses: the PRISMA statement. PLoS Med. 2009;6(7), e1000097. https://doi.org/10.1371/journal.pmed1000097.

21 Mahat RK, Panda S, Rathore V, Swain S, Yadav L, Sah SP. The dynamics of inflammatory markers in coronavirus disease-2019 (COVID-19) patients: a systematic review and meta-analysis. PROSPERO 2020 CRD42020193169 Available from: https://www.crd.york.ac.uk/prospero/display_record.php? ID $=$ CRD42020193169.

22 Wells G, Shea B, O'Connell D, et al. The Newcastle-Ottawa Scale (NOS) for assessing the quality of nonrandomised studies in meta-analyses. http://www.ohri.ca/progr ams/clinical_epidemiology/oxford.asp.

23 Luo D, Wan X, Liu J, Tong T. Optimally estimating the sample mean from the sample size, median, mid-range, and/or mid-quartile range. Stat Methods Med Res. 2018;27(6):1785-1805. https://doi.org/10.1177/0962280216669183.

24 Wan X, Wang W, Liu J, Tong T. Estimating the sample mean and standard deviation from the sample size, median, range and/or interquartile range. BMC Med Res Methodol. 2014;14:135. https://doi.org/10.1186/1471-2288-14-135.

25 Duval S, Tweedie R. Trim and fill: a simple funnel-plot-based method of testing and adjusting for publication bias in meta-analysis. Biometrics. 2000;56(2):455-463. https://doi.org/10.1111/j.0006-341x.2000.00455.x.

26 Cao Z, Li T, Liang L, et al. Clinical characteristics of coronavirus disease 2019 patients in Beijing, China. PloS One. 2020;15(6), e0234764. https://doi.org/ 10.1371/journal.pone.0234764.

27 Cen Y, Chen X, Shen Y, et al. Risk factors for disease progression in patients with mild to moderate coronavirus disease 2019-a multicentre observational study. Clin
Microbiol Infect. 2020 June 9. https://doi.org/10.1016/j.cmi.2020.05.041 [online ahead of print].

28 Chen C, Wang H, Liang Z, et al. Predicting illness severity and short-term outcomes of COVID-19: a retrospective cohort study in China. Innovation. 2020 May 21;1(1): 100007. https://doi.org/10.1016/j.xinn.2020.04.007.

29 Chen Q, Zheng Z, Zhang C, et al. Clinical characteristics of 145 patients with corona virus disease 2019 (COVID-19) in Taizhou, Zhejiang, China. Infection. 2020 Apr 28. https://doi.org/10.1007/s15010-020-01432-5 [online ahead of print].

30 Chen X, Zhao B, Qu Y, et al. Detectable serum SARS-CoV-2 viral load (RNAaemia) is closely correlated with drastically elevated interleukin 6 (IL-6) level in critically ill COVID-19 patients [published online ahead of print, 2020 Apr 17]. Clin Infect Dis. 2020:ciaa449. https://doi.org/10.1093/cid/ciaa449.

31 Ding X, Yu Y, Lu B, et al. Dynamic profile and clinical implications of hematological parameters in hospitalized patients with coronavirus disease 2019. Clin Chem Lab Med. 2020 May 22. https://doi.org/10.1515/cclm-2020-0411 (ahead-of-print).

32 Dong Y, Zhou H, Li M, et al. A novel simple scoring model for predicting severity of patients with SARS-CoV-2 infection [published online ahead of print, 2020 May 29]. Transbound Emerg Dis. 2020. https://doi.org/10.1111/tbed.13651, 10.1111/ tbed.13651.

33 Dreher M, Kersten A, Bickenbach J, et al. The characteristics of 50 hospitalized COVID-19 patients with and without ARDS. Dtsch Arztebl Int. 2020;117(16): 271-278. https://doi.org/10.3238/arztebl.2020.0271.

34 Duan J, Wang X, Chi J, et al. Correlation between the variables collected at admission and progression to severe cases during hospitalization among patients with COVID-19 in Chongqing [published online ahead of print, 2020 May 29]. J Med Virol. 2020. https://doi.org/10.1002/jmv.26082, 10.1002/jmv.26082.

35 Feng Y, Ling Y, Bai T, et al. COVID-19 with different severities: a multicenter study of clinical features. Am J Respir Crit Care Med. 2020;201(11):1380-1388. https:// doi.org/10.1164/rccm.202002-0445OC.

36 Fu J, Kong J, Wang W, et al. The clinical implication of dynamic neutrophil to lymphocyte ratio and D-dimer in COVID-19: a retrospective study in Suzhou China [published online ahead of print, 2020 May 6]. Thromb Res. 2020. https://doi.org/ 10.1016/j.thromres.2020.05.006.

37 Gao Y, Li T, Han M, et al. Diagnostic utility of clinical laboratory data determinations for patients with the severe COVID-19 [published online ahead of print, 2020 March 17]. J Med Virol. 2020. https://doi.org/10.1002/jmv.25770.

38 Gong J, Ou J, Qiu X, et al. A tool for early prediction of severe coronavirus disease 2019 (COVID-19): a multicenter study using the risk nomogram in Wuhan and Guangdong, China [published online ahead of print, 2020 April 16]. Clin Infect Dis. 2020. https://doi.org/10.1093/cid/ciaa443.

39 Huang C, Wang Y, Li X, et al. Clinical features of patients infected with 2019 novel coronavirus in Wuhan, China. Lancet. 2020;395(10223):497-506. https://doi.org/ 10.1016/S0140-6736(20)30183-5.

40 Jang JG, Hur J, Choi EY, Hong KS, Lee W, Ahn JH. Prognostic factors for severe coronavirus disease 2019 in Daegu, Korea. J Kor Med Sci. 2020;35(23), e209. https://doi.org/10.3346/jkms.2020.35.e209.

41 Khamis F, Al-Zakwani I, Al Naamani H, et al. Clinical characteristics and outcomes of the first 63 adult patients hospitalized with COVID-19: an experience from Oman. J Infect Public Health. 2020. https://doi.org/10.1016/j.jiph.2020.06.002 [Available online on June 8].

42 Lagadinou M, Salomou EE, Zareifopoulos N, Marangos M, Gogos C, Velissaris D. Prognosis of COVID-19: changes in laboratory parameters. Inf Med. 2020;28(suppl 1):89-95.

43 Li H, Xiang X, Ren H, et al. Serum Amyloid A is a biomarker of severe Coronavirus Disease and poor prognosis. J Infect. 2020;80(6):646-655. https://doi.org/ 10.1016/j.jinf.2020.03.035.

$44 \mathrm{Li} \mathrm{J}$, Li M, Zheng S, et al. Plasma albumin levels predict risk for nonsurvivors in critically ill patients with COVID-19 [published online ahead of print, 2020 Jun 3]. Biomarkers Med. 2020. https://doi.org/10.2217/bmm-2020-0254, 10.2217/bmm2020-0254.

$45 \mathrm{Li} \mathrm{K}, \mathrm{Wu}$ J, Wu F, et al. The clinical and chest CT features associated with severe and critical COVID-19 pneumonia. Invest Radiol. 2020;55(6):327-331. https://doi.org/ 10.1097/RLI.0000000000000672.

46 Liu J, Li S, Liu J, et al. Longitudinal characteristics of lymphocyte responses and cytokine profiles in the peripheral blood of SARS-CoV-2 infected patients. EBioMedicine. 2020;55:102763. https://doi.org/10.1016/j.ebiom.2020.102763.

47 Liu T, Zhang J, Yang Y, et al. The role of interleukin-6 in monitoring severe case of coronavirus disease 2019. EMBO Mol Med. 2020. https://doi.org/10.15252/ emmm.202012421 [Available online on June 5] doi:.

48 Lo IL, Lio CF, Cheong HH, et al. Evaluation of SARS-CoV-2 RNA shedding in clinical specimens and clinical characteristics of 10 patients with COVID-19 in Macau. Int $J$ Biol Sci. 2020;16(10):1698-1707. https://doi.org/10.7150/ijbs.45357.

49 Ma J, Yin J, Qian Y, Wu Y. Clinical characteristics and prognosis in cancer patients with COVID-19: a single center's retrospective study. J Infect. 2020. https://doi. org/10.1016/j.jinf.2020.04.006 [Available online on April 14] doi:.

50 Ortiz-Brizuela E, Villanueva-Reza M, González-Lara MF, et al. Clinical and epidemiological characteristics of patients diagnosed with COVID-19 in a tertiary care center in Mexico City: a prospective cohort study. Rev Invest Clin. 2020;72(3): 165-177. https://doi.org/10.24875/RIC.20000211.

51 Shahriarirad R, Khodamoradi Z, Erfani A, et al. Epidemiological and clinical features of 2019 novel coronavirus diseases (COVID-19) in the South of Iran. BMC Infect Dis. 2020;20(1):427. https://doi.org/10.1186/s12879-020-05128-x.

52 Shang W, Dong J, Ren Y, et al. The value of clinical parameters in predicting the severity of COVID-19 [published online ahead of print, 2020 May 21]. J Med Virol. 2020. https://doi.org/10.1002/jmv.26031, 10.1002/jmv.26031. 
53 Shao L, Li X, Zhou Y, et al. Novel insights into illness progression and risk profiles for mortality in non-survivors of COVID-19. Front Med. 2020;7:246. https://doi. org/10.3389/fmed.2020.00246.

54 Shi J, Li Y, Zhou X, et al. Lactate dehydrogenase and susceptibility to deterioration of mild COVID-19 patients: a multicenter nested case-control study. BMC Med. 2020;18(1):168. https://doi.org/10.1186/s12916-020-01633-7.

55 Spinetti T, Hirzel C, Fux M, et al. Reduced monocytic HLA-DR expression indicates immunosuppression in critically ill COVID-19 patients [published online ahead of print, 2020 Jun 4]. Anesth Analg. 2020. https://doi.org/10.1213/ ANE.0000000000005044, 10.1213/ANE.0000000000005044.

56 Sun Y, Dong Y, Wang L, et al. Characteristics and prognostic factors of disease severity in patients with COVID-19: the Beijing experience. $J$ Autoimmun. 2020. https://doi.org/10.1016/j.jaut.2020.102473 [Available online on April 24].

57 Tian J, Yuan X, Xiao J, et al. Clinical characteristics and risk factors associated with COVID-19 disease severity in patients with cancer in Wuhan, China: a multicentre, retrospective, cohort study. Lancet Oncol. 2020. https://doi.org/10.1016/S14702045(20)30309-0 [Available online on May 29].

58 Wan S, Yi Q, Fan S, et al. Relationships among lymphocyte subsets, cytokines, and the pulmonary inflammation index in coronavirus (COVID-19) infected patients. $\mathrm{Br}$ J Haematol. 2020;189(3):428-437. https://doi.org/10.1111/bjh.16659.

59 Wang $\mathrm{G}$, Wu C, Zhang Q, et al. C-reactive protein level may predict the risk of COVID-19 aggravation. Open Forum Infect Dis. 2020;7(5). https://doi.org/10.1093/ ofid/ofaa153. ofaa153.

60 Wang L. C-reactive protein levels in the early stage of COVID-19. Med Maladies Infect. 2020;50(4):332-334. https://doi.org/10.1016/j.medmal.2020.03.007.

61 Wang R, Pan M, Zhang X, et al. Epidemiological and clinical features of 125 hospitalized patients with COVID-19 in Fuyang, Anhui, China. Int J Infect Dis. 2020; 95:421-428. https://doi.org/10.1016/j.ijid.2020.03.070.

62 Wang Y, Liao B, Guo Y, et al. Clinical characteristics of patients infected with the novel 2019 coronavirus (SARS-Cov-2) in Guangzhou, China. Open Forum Infect Dis. 2020;7(6):ofaa187. https://doi.org/10.1093/ofid/ofaa187.

63 Wang Z, Yang B, Li Q, Wen L, Zhang R. Clinical features of 69 cases with coronavirus disease 2019 in Wuhan, China. Clin Infect Dis. 2020. https://doi.org/ 10.1093/cid/ciaa272 [Published online on March 16].

64 Wei X, Su J, Yang K, et al. Elevations of serum cancer biomarkers correlate with severity of COVID-19 [published online ahead of print on Apr 29]. J Med Virol. 2020. https://doi.org/10.1002/jmv.25957, 10.1002/jmv.25957.

65 Wei X, Zeng W, Su J, et al. Hypolipidemia is associated with the severity of COVID19. J Clin Lipidol. 2020;14(3):297-304. https://doi.org/10.1016/j. jacl.2020.04.008.

$66 \mathrm{Wu} \mathrm{C}$, Chen X, Cai Y, et al. Risk factors associated with acute respiratory distress syndrome and death in patients with coronavirus disease 2019 pneumonia in Wuhan, China. JAMA Intern Med. 2020. https://doi.org/10.1001/ jamainternmed.2020.0994 [Published online on March 13].

67 Xie H, Zhao J, Lian N, Lin S, Xie Q, Zhuo H. Clinical characteristics of non-ICU hospitalized patients with coronavirus disease 2019 and liver injury: a retrospective study. Liver Int. 2020;40(6):1321-1326. https://doi.org/10.1111/liv.14449.

$68 \mathrm{Xu} \mathrm{B}$, Fan CY, Wang AL, et al. Suppressed T cell-mediated immunity in patients with COVID-19: a clinical retrospective study in Wuhan, China. J Infect. 2020. https:// doi.org/10.1016/j.jinf.2020.04.012 [Published online on April 18].

69 Yang A, Qiu Q, Kong X, et al. Clinical and epidemiological characteristics of COVID19 patients in Chongqing China. Front Public Health. 2020;8:244. https://doi.org/ 10.3389/fpubh.2020.00244.

70 Yang AP, Liu JP, Tao WQ, Li HM. The diagnostic and predictive role of NLR, d-NLR and PLR in COVID-19 patients. Int Immunopharm. 2020;84:106504. https://doi.org/ 10.1016/j.intimp.2020.106504.

71 Yang L, Liu J, Zhang R, et al. Epidemiological and clinical features of 200 hospitalized patients with corona virus disease 2019 outside Wuhan, China: a descriptive study. J Clin Virol. 2020;129:104475. https://doi.org/10.1016/j. jcv.2020.104475.

72 Yang Q, Xie L, Zhang W, et al. Analysis of the clinical characteristics, drug treatments and prognoses of 136 patients with coronavirus disease 2019. J Clin Pharm Therapeut. 2020:1-8. https://doi.org/10.1111/jcpt.13170.

73 Yao $\mathrm{Q}$, Wang $\mathrm{P}$, Wang $\mathrm{X}$, et al. A retrospective study of risk factors for severe acute respiratory syndrome coronavirus 2 infections in hospitalized adult patients. Pol Arch Intern Med. 2020;130(5):390-399. https://doi.org/10.20452/pamw.15312.

74 Young BE, Ong SWX, Kalimuddin S, et al. Epidemiologic features and clinical course of patients infected with SARS-CoV-2 in Singapore. J Am Med Assoc. 2020; 323(15):1488-1494. https://doi.org/10.1001/jama.2020.3204.

75 Zeng QL, Li GM, Ji F, et al. Clinical course and treatment efficacy of COVID-19 near Hubei Province, China: a multicentre, retrospective study [published online ahead of print, 2020 Jun 12]. Transbound Emerg Dis. 2020. https://doi.org/10.1111/ tbed.13674, 10.1111/tbed.13674.

76 Zhang JJ, Dong X, Cao YY, et al. Clinical characteristics of 140 patients infected with SARS-CoV-2 in Wuhan, China. Allergy. 2020:1-12. https://doi.org/10.1111/ all.14238.

77 Zhang Y, Zheng L, Liu L, Zhao M, Xiao J, Zhao Q. Liver impairment in COVID-19 patients: a retrospective analysis of 115 cases from a single centre in Wuhan city, China [published online ahead of print, 2020 Apr 2]. Liver Int. 2020. https://doi. org/10.1111/liv.14455, 10.1111/liv.14455.

78 Zheng F, Tang W, Li H, Huang YX, Xie YL, Zhou ZG. Clinical characteristics of 161 cases of corona virus disease 2019 (COVID-19) in Changsha. Eur Rev Med Pharmacol Sci. 2020;24(6):3404-3410. https://doi.org/10.26355/eurrev_202003_20711.

79 Zheng Y, Xu H, Yang M, et al. Epidemiological characteristics and clinical features of 32 critical and 67 noncritical cases of COVID-19 in Chengdu. J Clin Virol. 2020 127:104366. https://doi.org/10.1016/j.jcv.2020.104366.
80 Zhu Z, Cai T, Fan L, et al. Clinical value of immune-inflammatory parameters to assess the severity of coronavirus disease 2019. Int J Infect Dis. 2020;95:332-339. https://doi.org/10.1016/j.ijid.2020.04.041.

81 Bonetti G, Manelli F, Patroni A, et al. Laboratory predictors of death from coronavirus disease 2019 (COVID-19) in the area of Valcamonica, Italy. Clin Chem Lab Med. 2020 Apr 28. https://doi.org/10.1515/cclm-2020-0459, 1(ahead-ofprint).

82 Chen T, Wu D, Chen H, et al. Clinical characteristics of 113 deceased patients with coronavirus disease 2019: retrospective study. BMJ. 2020;368:m1091. https://doi. org/10.1136/bmj.m1091.

83 Chen TL, Dai Z, Mo P, et al. Clinical characteristics and outcomes of older patients with coronavirus disease 2019 (COVID-19) in Wuhan, China (2019): a singlecentered, retrospective study. J Gerontol A Biol Sci Med Sci. 2020 Apr 11. https:// doi.org/10.1093/gerona/glaa089. glaa089.

84 Covino M, De Matteis G, Santoro M, et al. Clinical characteristics and prognostic factors in COVID-19 patients aged $\geq 80$ years. Geriatr Gerontol Int. 2020 Jul;20(7): 704-708. https://doi.org/10.1111/ggi.13960.

85 Deng Y, Liu W, Liu K, et al. Clinical characteristics of fatal and recovered cases of coronavirus disease 2019 in Wuhan, China: a retrospective study. Chin Med J. 2020 Jun 5;133(11):1261-1267. https://doi.org/10.1097/CM9.0000000000000824.

86 Giacomelli A, Ridolfo AL, Milazzo L, et al. 30-day mortality in patients hospitalized with COVID-19 during the first wave of the Italian epidemic: a prospective cohort study. Pharmacol Res 2020:104931. doi:10.1016/j.phrs.2020.104931.

87 Huang J, Cheng A, Kumar R, et al. Hypoalbuminemia predicts the outcome of COVID-19 independent of age and co-morbidity. J Med Virol. 2020 May 14. https:// doi.org/10.1002/jmv.26003.

88 Javanian M, Bayani M, Shokri M, et al. Clinical and laboratory findings from patients with COVID-19 pneumonia in Babol North of Iran: a retrospective cohort study [published online ahead of print, 2020 May 11]. Rom J Intern Med; 2020. http://j/rjim.ahead-of-print/rjim-2020-0013/rjim-2020-0013.xml. doi:10. 2478/rjim-2020-0013.

89 Lee JY, Kim HA, Huh K, et al. Risk factors for mortality and respiratory support in elderly patients hospitalized with COVID-19 in Korea. J Kor Med Sci. 2020 Jun 15; 35(23):e223. https://doi.org/10.3346/jkms.2020.35.e223.

$90 \mathrm{Li}$ L, Yang L, Gui S, et al. Association of clinical and radiographic findings with the outcomes of 93 patients with COVID-19 in Wuhan, China. Theranostics. 2020 May 15;10(14):6113-6121. https://doi.org/10.7150/thno.46569.

91 Luo X, Zhou W, Yan X, et al. Prognostic value of C-reactive protein in patients with COVID-19 [published online ahead of print, 2020 May 23]. Clin Infect Dis. 2020. https://doi.org/10.1093/cid/ciaa641. ciaa641.

92 Masetti C, Generali E, Colapietro F, et al. High mortality in COVID-19 patients with mild respiratory disease. Eur J Clin Invest. 2020 Jun 14, e13314. https://doi.org/ 10.1111/eci.13314.

93 Pan F, Yang L, Li Y, et al. Factors associated with death outcome in patients with severe coronavirus disease-19 (COVID-19): a case-control study. Int J Med Sci. 2020 May 18;17(9):1281-1292. https://doi.org/10.7150/ijms.46614.

94 Ruan Q, Yang K, Wang W, Jiang L, Song J. Clinical predictors of mortality due to COVID-19 based on an analysis of data of 150 patients from Wuhan, China [published correction appears in Intensive Care Med. Intensive Care Med. 2020;46 (5):846-848. https://doi.org/10.1007/s00134-020-05991-x, 2020 Apr 6.

95 Satici C, Demirkol MA, Altunok ES, et al. Performance of pneumonia severity index and CURB-65 in predicting 30-day mortality in patients with COVID-19. Int $J$ Infect Dis. 2020. https://doi.org/10.1016/j.ijid.2020.06.038.

96 Shi S, Qin M, Cai Y, et al. Characteristics and clinical significance of myocardial injury in patients with severe coronavirus disease 2019. Eur Heart J. 2020;41(22): 2070-2079. https://doi.org/10.1093/eurheartj/ehaa408.

97 Sun H, Ning R, Tao Y, et al. Risk factors for mortality in 244 older adults with COVID-19 in Wuhan, China: a retrospective study. J Am Geriatr Soc. 2020 Jun;68 (6):E19-E23. https://doi.org/10.1111/jgs.16533.

98 Tomlins J, Hamilton F, Gunning S, Sheehy C, Moran E, MacGowan A. Clinical features of 95 sequential hospitalised patients with novel coronavirus 2019 disease (COVID-19), the first UK cohort. J Infect. 2020. https://doi.org/10.1016/j. jinf.2020.04.020 [Published online on April 27].

99 Tu WJ, Cao J, Yu L, Hu X, Liu Q. Clinicolaboratory study of 25 fatal cases of COVID19 in Wuhan. Intensive Care Med. 2020 Jun;46(6):1117-1120. https://doi.org/ 10.1007/s00134-020-06023-4.

100 Wang L, He W, Yu X, et al. Coronavirus disease 2019 in elderly patients: characteristics and prognostic factors based on 4-week follow-up. J Infect. 2020 Jun; 80(6):639-645. https://doi.org/10.1016/j.jinf.2020.03.019.

101 Yan X, Li F, Wang X, et al. Neutrophil to lymphocyte ratio as prognostic and predictive factor in patients with coronavirus disease 2019: a retrospective crosssectional study. J Med Virol. 2020 May 26. https://doi.org/10.1002/jmv.26061.

102 Yang K, Sheng Y, Huang C, et al. Clinical characteristics, outcomes, and risk factors for mortality in patients with cancer and COVID-19 in Hubei, China: a multicentre, retrospective, cohort study. Lancet Oncol. 2020 May 29. https://doi.org/10.1016/ S1470-2045(20)30310-7 [Epub ahead of print].

103 Zhang N, Xu X, Zhou LY, et al. Clinical characteristics and chest CT imaging features of critically ill COVID-19 patients. Eur Radiol. 2020 May 30:1-10. https://doi.org/ 10.1007/s00330-020-06955-x.

104 Zhou F, Yu T, Du R, et al. Clinical course and risk factors for mortality of adult inpatients with COVID-19 in Wuhan, China: a retrospective cohort study. Lancet. 2020;28(10229):1054-1062. https://doi.org/10.1016/S0140-6736(20)30566-3, 395.

105 Black S, Kushner I, Samols D. C-reactive protein. J Biol Chem. 2004;279(47): 48487-48490. https://doi.org/10.1074/jbc.R400025200. 
106 Chalmers S, Khawaja A, Wieruszewski PM, Gajic O, Odeyemi Y. Diagnosis and treatment of acute pulmonary inflammation in critically ill patients: the role of inflammatory biomarkers. World J Crit Care Med. 2019;8(5):59-71. https://doi.org/ 10.5492/wjccm.v8.i5.59.

107 Khalid I, Alraddadi BM, Dairi Y, et al. Acute management and long-term survival among subjects with severe Middle East respiratory syndrome coronavirus pneumonia and ARDS. Respir Care. 2016;61(3):340-348. https://doi.org/10.4187/ respcare. 04325 .

108 Cheng ZJ, Shan J. Novel coronavirus: where we are and what we know. Infection. 2019;48(2):155-163. https://doi.org/10.1007/s15010-020-01401-y, 2020.

$109 \mathrm{Wu}$ S, Zhou Y, Hua HY, et al. Inflammation marker ESR is effective in predicting outcome of diffuse large B-cell lymphoma. BMC Canc. 2018;18(1):997. https://doi. org/10.1186/s12885-018-4914-4.

110 Lapić I, Rogić D, Plebani M. Erythrocyte sedimentation rate is associated with severe coronavirus disease 2019 (COVID-19): a pooled analysis. Clin Chem Lab Med. 2020;58(7):1146-1148. https://doi.org/10.1515/cclm-2020-0620.

111 Henry BM, de Oliveira MHS, Benoit S, Plebani M, Lippi G. Hematologic, biochemical and immune biomarker abnormalities associated with severe illness and mortality in coronavirus disease 2019 (COVID-19): a meta-analysis. Clin Chem Lab Med. 2020;58(7):1021-1028. https://doi.org/10.1515/cclm-2020-0369.

112 Lippi G, Cervellin G. Procalcitonin for diagnosing and monitoring bacterial infections: for or against? Clin Chem Lab Med. 2018;56(8):1193-1195. https://doi org/10.1515/cclm-2018-0312.

113 Creamer AW, Kent AE, Albur M. Procalcitonin in respiratory disease: use as a biomarker for diagnosis and guiding antibiotic therapy. Breathe. 2019;15(4): 296-304. https://doi.org/10.1183/20734735.0258-2019.

114 Song J, Park DW, Moon S, et al. Diagnostic and prognostic value of interleukin-6, pentraxin 3 , and procalcitonin levels among sepsis and septic shock patients: a prospective controlled study according to the Sepsis-3 definitions. BMC Infect Dis. 2019;19(1):968. https://doi.org/10.1186/s12879-019-4618-7.

115 Lippi G, Plebani M. Procalcitonin in patients with severe coronavirus disease 2019 (COVID-19): a meta-analysis. Clin Chim Acta. 2020;505:190-191. https://doi.org/ 10.1016/j.cca.2020.03.004.

116 Schuetz P, Albrich W, Mueller B. Procalcitonin for diagnosis of infection and guide to antibiotic decisions: past, present and future. BMC Med. 2011;9:107. https://doi. org/10.1186/1741-7015-9-107.

117 Chollet-Martin S, Jourdain B, Gibert C, Elbim C, Chastre J, Gougerot-Pocidalo MA Interactions between neutrophils and cytokines in blood and alveolar spaces during ARDS. Am J Respir Crit Care Med. 1996;154(3 Pt 1):594-601. https://doi.org/ 10.1164/ajrccm.154.3.8810592.

118 Gupta KK, Khan MA, Singh SK. Constitutive inflammatory cytokine storm: a major threat to human Health. J Interferon Cytokine Res. 2020;40(1):19-23. https://doi. org/10.1089/jir.2019.0085.

119 Tanaka T, Narazaki M, Kishimoto T. IL-6 in inflammation, immunity, and disease. Cold Spring Harb Perspect Biol. 2014;6(10):a016295. https://doi.org/10.1101/ cshperspect.a016295.

120 Tanaka T, Narazaki M, Kishimoto T. Immunotherapeutic implications of IL-6 blockade for cytokine storm. Immunotherapy. 2016;8(8):959-970. https://doi.org/ 10.2217/imt-2016-0020.

121 Jose RJ, Manuel A. COVID-19 cytokine storm: the interplay between inflammation and coagulation. Lancet Respir Med. 2020;8(6):e46-e47. https://doi.org/10.1016/ S2213-2600(20)30216-2.

122 Chen G, Wu D, Guo W, et al. Clinical and immunological features of severe and moderate coronavirus disease 2019. J Clin Invest. 2020;130(5):2620-2629. https:// doi.org/10.1172/JCI137244.

123 Channappanavar R, Fehr AR, Vijay R, et al. Dysregulated type I interferon and inflammatory monocyte-macrophage responses cause lethal pneumonia in SARSCoV-infected mice. Cell Host Microbe. 2016;19(2):181-193. https://doi.org/ 10.1016/j.chom.2016.01.007.
124 Cameron MJ, Bermejo-Martin JF, Danesh A, Muller MP, Kelvin DJ. Human immunopathogenesis of severe acute respiratory syndrome (SARS). Virus Res. 2008; 133(1):13-19. https://doi.org/10.1016/j.virusres.2007.02.014.

125 Wong CK, Lam CW, Wu AK, et al. Plasma inflammatory cytokines and chemokines in severe acute respiratory syndrome. Clin Exp Immunol. 2004;136(1):95-103. https://doi.org/10.1111/j.1365-2249.2004.02415.x.

126 Min CK, Cheon S, Ha NY, et al. Comparative and kinetic analysis of viral shedding and immunological responses in MERS patients representing a broad spectrum of disease severity. Sci Rep. 2016;6:25359. https://doi.org/10.1038/srep25359.

127 Xu X, Han M, Li T, et al. Effective treatment of severe COVID-19 patients with tocilizumab. Proc Natl Acad Sci U S A. 2020;117(20):10970-10975. https://doi.org/ 10.1073/pnas. 2005615117.

128 Bachmann MF, Oxenius A. Interleukin 2: from immunostimulation to immunoregulation and back again. EMBO Rep. 2007;8(12):1142-1148. https://doi. org/10.1038/sj.embor.7401099.

129 Nie Z, Scott GD, Weis PD, Itakura A, Fryer AD, Jacoby DB. Role of TNF- $\alpha$ in virusinduced airway hyperresponsiveness and neuronal $\mathrm{M}_{2}$ muscarinic receptor dysfunction. Br J Pharmacol. 2011;164(2b):444-452. https://doi.org/10.1111/ j.1476-5381.2011.01393.x.

130 Kernan KF, Carcillo JA. Hyperferritinemia and inflammation. Int Immunol. 2017;29 (9):401-409. https://doi.org/10.1093/intimm/dxx031.

131 Mehta P, McAuley DF, Brown M, et al. COVID-19: consider cytokine storm syndromes and immunosuppression. Lancet. 2020;395(10229):1033-1034. https:// doi.org/10.1016/S0140-6736(20)30628-0.

132 Baranova IN, Souza ACP, Bocharov AV, et al. Human SR-BII mediates SAA uptake and contributes to SAA pro-inflammatory signaling in vitro and in vivo. PloS One. 2017;12(4), e0175824. https://doi.org/10.1371/journal.pone.0175824.

133 Ye RD, Sun L. Emerging functions of serum amyloid A in inflammation. J Leukoc Biol. 2015;98(6):923-929. https://doi.org/10.1189/jlb.3VMR0315-080R.

134 Yang RZ, Lee MJ, Hu H, et al. Acute-phase serum amyloid A: an inflammatory adipokine and potential link between obesity and its metabolic complications. PLoS Med. 2006;3(6):e287. https://doi.org/10.1371/journal.pmed.0030287.

135 De Buck M, Gouwy M, Wang JM, et al. Structure and expression of different serum amyloid A (SAA) variants and their concentration-dependent functions during host insults. Curr Med Chem. 2016;23(17):1725-1755. https://doi.org/10.2174/ 0929867323666160418114600.

136 Connolly M, Rooney PR, McGarry T, et al. Acute serum amyloid A is an endogenous TLR2 ligand that mediates inflammatory and angiogenic mechanisms. Ann Rheum Dis. 2016;75(7):1392-1398. https://doi.org/10.1136/annrheumdis-2015-207655.

137 Sack Jr GH. Serum amyloid A - a review. Mol Med. 2018;24(1):46. https://doi.org/ 10.1186/s10020-018-0047-0.

138 Yip TT, Chan JW, Cho WC, et al. Protein chip array profiling analysis in patients with severe acute respiratory syndrome identified serum amyloid A protein as a biomarker potentially useful in monitoring the extent of pneumonia. Clin Chem. 2005;51(1):47-55. https://doi.org/10.1373/clinchem.2004.031229.

139 Behrens K, Alexander WS. Cytokine control of megakaryopoiesis. Growth Factors. 2018;36(3-4):89-103. https://doi.org/10.1080/08977194.2018.1498487.

140 Meng LB, Yu ZM, Guo P, et al. Neutrophils and neutrophil-lymphocyte ratio: inflammatory markers associated with intimal-media thickness of atherosclerosis. Thromb Res. 2018;170:45-52. https://doi.org/10.1016/j.thromres.2018.08.002.

141 Wang D, Hu B, Hu C, et al. Clinical characteristics of 138 hospitalized patients with 2019 novel coronavirus-infected pneumonia in Wuhan, China. J Am Med Assoc. 2020;323(11):1061-1069. https://doi.org/10.1001/jama.2020.1585.

142 Lagunas-Rangel FA. Neutrophil-to-lymphocyte ratio and lymphocyte-to-C-reactive protein ratio in patients with severe coronavirus disease 2019 (COVID-19): a metaanalysis [published online ahead of print, 2020 Apr 3]. J Med Virol. 2020. https:// doi.org/10.1002/jmv.25819. 